\title{
A Smad3-PTEN regulatory loop controls proliferation and apoptotic responses to TGF- $\beta$ in mouse endometrium
}

Nuria Eritja ${ }^{1,2}$, Isidre Felip ${ }^{1}$, Mari Alba Dosil ${ }^{1,2}$, Lucia Vigezzi ${ }^{3}$, Cristina Mirantes ${ }^{1}$, Andree Yeramian ${ }^{1,2}$, Raúl Navaridas $^{1}$, Maria Santacana $^{1,2}$, David Llobet-Navas ${ }^{4}$, Akihiko Yoshimura ${ }^{5}$, Masatoshi Nomura ${ }^{6}$, Mario Encinas ${ }^{7}$, Xavier Matias-Guiu $^{1,2}$ and Xavi Dolcet, ${ }^{\star, 1,2}$

The TGF- $\beta /$ Smad and the PI3K/AKT signaling pathways are important regulators of proliferation and apoptosis, and their alterations lead to cancer development. TGF- $\beta$ acts as a tumor suppressor in premalignant cells, but it is a tumor promoter for cancerous cells. Such dichotomous actions are dictated by different cellular contexts. Here, we have unveiled a PTEN-Smad3 regulatory loop that provides a new insight in the complex cross talk between TGF- $\beta /$ Smad and PI3K/AKT signaling pathways. We demonstrate that TGF- $\beta$ triggers apoptosis of wild-type polarized endometrial epithelial cells by a Smad3-dependent activation of PTEN transcription, which results in the inhibition of PI3K/AKT signaling pathway. We show that specific Smad3 knockdown or knockout reduces basal and TGF- $\beta$-induced PTEN expression in endometrial cells, resulting in a blockade of TGF- $\beta$-induced apoptosis and an enhancement of cell proliferation. Likewise Smad3 deletion, PTEN knockout prevents TGF- $\beta$-induced apoptosis and increases cell proliferation by increasing PI3K/AKT/mTOR signaling. In summary, our results demonstrate that Smad3-PTEN signaling axis determine cellular responses to TGF- $\beta$.

Cell Death and Differentiation (2017) 24, 1443-1458; doi:10.1038/cdd.2017.73; published online 19 May 2017

TGF- $\beta$ is a multifunctional cytokine involved in the regulation of a wide range of biological processes. ${ }^{1}$ TGF- $\beta$ signaling is initiated by ligand binding to the constitutively active dualspecificity kinase TGF- $\beta$ type II receptor (T $\beta$ RII). Engaged $T \beta R$ II interacts with the TGF- $\beta$ type I receptor (T $\beta R I$ ) to form tetrameric complexes. Then, T $\beta R$ II transphosphorylates T $\beta R I$ at the glycine-serine-rich domain. The canonical T $\beta R s$ signaling is conducted by the Smad family of transcription factors. ${ }^{2-4}$ Activation of T $\beta R$ complex results in the phosphorylation of the receptor-associated Smads (R-Smads), Smad2 and Smad3, which interact with the common Smad (Co-Smad) Smad4. Smad4-R-Smad complexes can bind other DNAbinding transcription factors to modulate target gene promoter recognition and transcriptional regulation. ${ }^{5}$ TGF- $\beta$ can also trigger other signaling pathways referred as 'non-Smad' branch of TGF- $\beta$ signaling ${ }^{6,7}$ such as the MAP kinase signaling pathway, the Rho-like GTPase signaling pathway or the phosphatidylinositol-3 kinase/AKT (PI3K/AKT) signaling pathway.

During carcinogenesis, TGF- $\beta$ is a double-edge sword: it acts as a potent tumor suppressor on normal or premalignant cells, but it has a tumor promoter activity on malignant stages. ${ }^{8-10}$ Tumor suppressive functions of TGF- $\beta$ depend on its ability to induce cytostasis, apoptosis and differentiation and, loss of such responses plays a pivotal role in cancer progression. ${ }^{9,11}$ Emerging data demonstrate that tumor suppressive signaling induced by TGF- $\beta$ is halted by oncogenic mutations. Among such alterations, those that activate the PI3K/AKT signaling pathway, antagonize cytostatic or pro-apoptotic effects of TGF- $\beta .^{12}$

The PI3K/AKT pathway plays a pivotal role in the regulation of cell survival and proliferation. PI3K/AKT signaling is negatively regulated by PTEN (phosphatase and tensin homolog deleted on chromosome 10), which encodes a dual lipid and protein phosphatase that antagonizes PI3K function by catalyzing the dephosphorylation of phosphatidylinositol3,4,5-trisphosphate (PIP3) to phosphatidylinositol-4,5-trisphosphate (PIP2). ${ }^{13}$ Alterations of PTEN increase PI3K/AKT signaling, which stimulates cell proliferation and survival.

Because both the TGF- $\beta /$ Smad and the PI3K/AKT signaling pathways participate in the control of similar cellular processes, a complex interplay between these two pathways is required to coordinate cellular outcomes. ${ }^{12}$ However, the mechanisms of cross talk between these two pathways are not completely elucidated and several models have been proposed. ${ }^{12}$ The first one demonstrates that AKT can directly interact and retain Smad3 in the cytoplasm and the transcription of target genes that drive apoptosis triggered by TGF- $\beta .{ }^{14,15} \mathrm{~A}$ second model demonstrates that AKT phosphorylates the forkhead transcription factor (FOXO) resulting

\footnotetext{
${ }^{1}$ Department de Ciències Mèdiques Bàsiques, Oncologic Pathology Group, Universitat de Lleida, Hospital Universitari Arnau de Vilanova, Institut de Recerca Biomèdica de Lleida, Lleida, Spain; ${ }^{2}$ Centro de Investigación Biomédica en Red de Oncología (CIBERONC), Madrid, Spain; ${ }^{3}$ Facultad de Bioquímica y Ciencias Biológicas, Instituto de Salud y Ambiente del Litoral (ISAL) - CONICET, Universidad Nacional del Litoral, Santa Fe, Argentina; ${ }^{4}$ Institute of Genetic Medicine, Newcastle University, Newcastle upon Tyne NE1 3BZ, UK; ${ }^{5}$ Department of Microbiology and Immunology, Keio University School of Medicine, 35 Shinanomachi, Tokyo 160-8582, Japan; ${ }^{6}$ Department of Medicine and Bioregulatory Science, Graduate School of Medical Science, Kyushu University, Maidashi, Fukuoka 812-8582, Japan and ${ }^{7}$ Department Medicina Experimental, Universitat de Lleida, Institut de Recerca Biomèdica de Lleida, Lleida, Spain

*Corresponding author: X Dolcet, Department de Ciències Mèdiques Bàsiques, Oncologic Pathology Group, Universitat de Lleida, Hospital Universitari Arnau de Vilanova, Institut de Recerca Biomèdica de Lleida, Hospital Arnau de Vilanova, Av Rovira Roure, 80, Lleida 25198, Spain. Tel: +34 973 702951; Email: dolcet@cmb.udl.cat Received 21.7.16; revised 24.3.17; accepted 05.4.17; Edited by S Fulda; published online 19.5.17
} 
on its nuclear export, thereby interfering the formation of a FOXO/Smad transcriptional complex, which is required for cytostatic responses to TGF- $\beta .^{16}$ A third line of evidence demonstrates that combination of PTEN loss and Smad4 inactivation leads to tumor progression in a mouse model of prostatic cancer. ${ }^{17,18}$ Recently, another study demonstrated that specific genetic deletion of T $\beta$ RII and PTEN Is increases intestinal tumorigenesis in mice. ${ }^{19}$
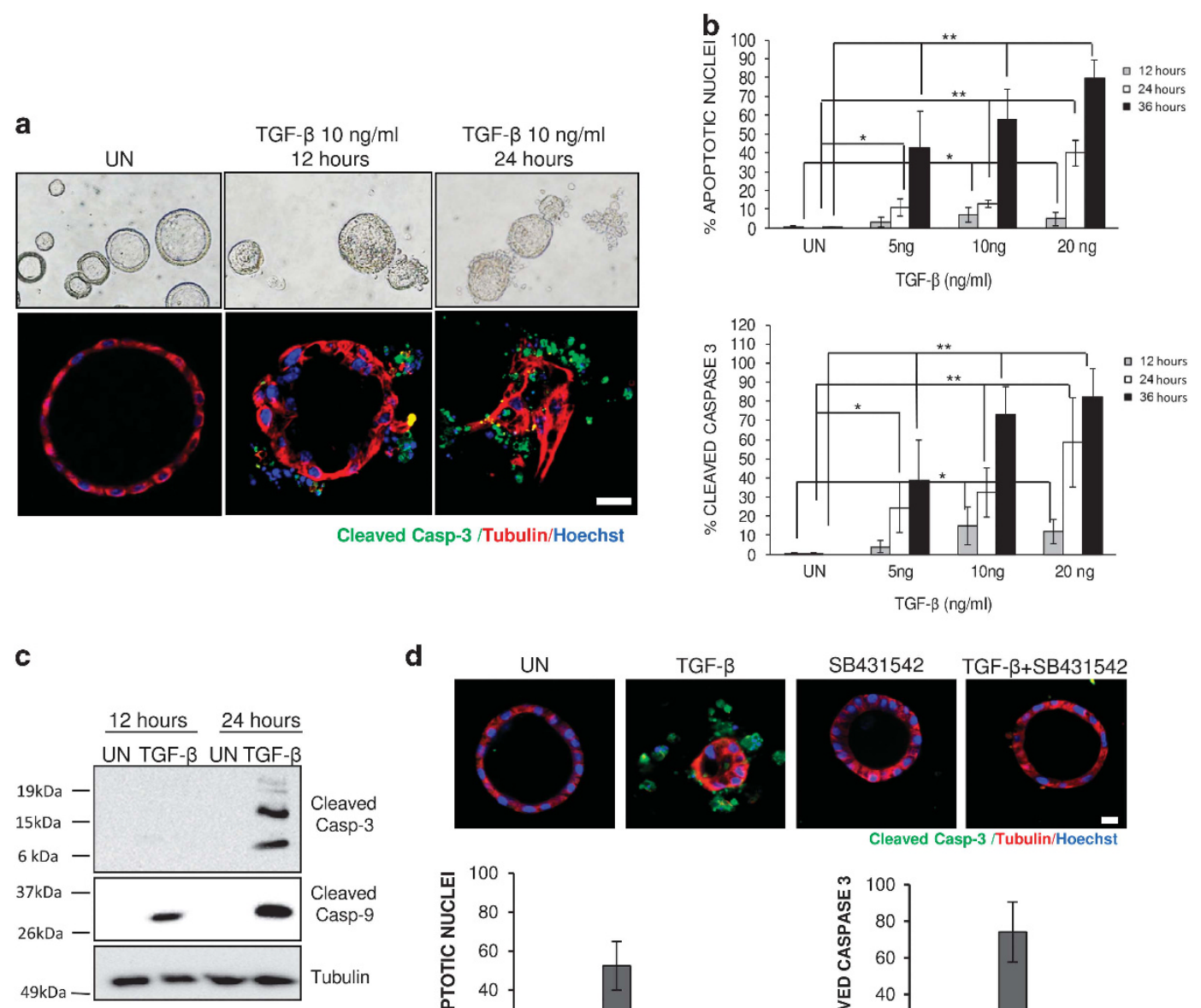

d
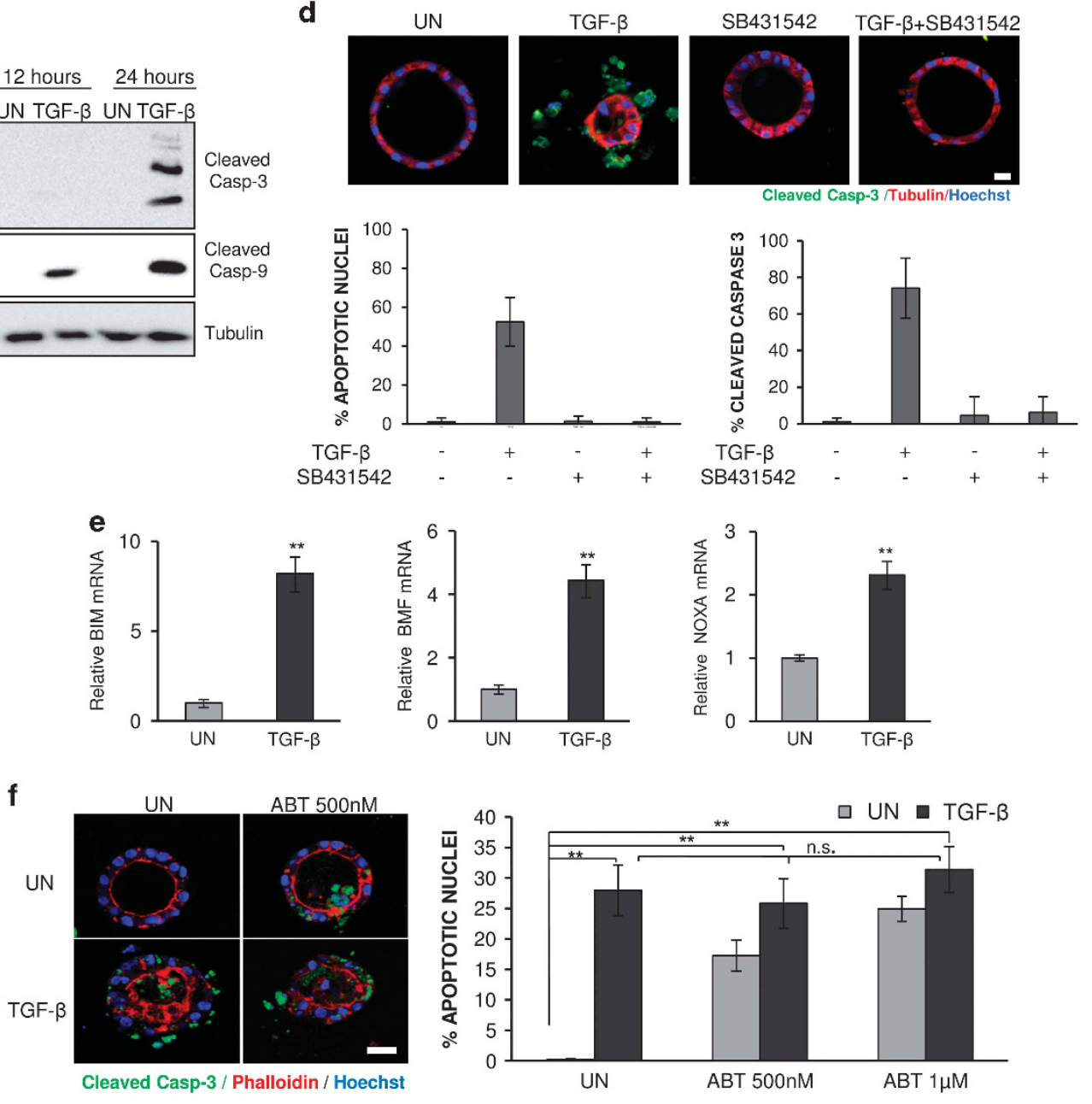
Endometrial carcinoma is the most common tumor of the female genital tract. Both PI3K/AKT and TGF- $\beta /$ Smad signaling pathways play a pivotal role in uterine function and physiology. ${ }^{20}$ Female mice lacking $T \beta R I$ in the reproductive tract exhibit profound defects in myometrium structure and function. ${ }^{21}$ Moreover, ablation of $\mathrm{T} \beta \mathrm{R} \mathrm{I}$ in the uterus increases endometrial cell proliferation causing endometrial hyperplasia, which often precedes endometrial carcinogenesis. ${ }^{22}$ Moreover, mutations in the PI3K/AKT signaling pathway are common in endometrial carcinogenesis. Among them, PTEN is the most frequently mutated tumor suppressor gene in endometrial carcinomas ${ }^{23}$ and genetic ablation of PTEN in mice results in a high incidence of endometrial carcinogenesis. ${ }^{24}$

Here, we describe a novel mechanism of interplay between PI3K/AKT and TGF- $\beta /$ Smad signaling pathways that plays a pivotal role in the regulation of cell proliferation and apoptosis of endometrial epithelial cells.

\section{Results}

TGF- $\beta$ triggers apoptosis of polarized endometrial epithelial cells. The effects of TGF- $\beta$ on uterine endometrial epithelial cells under physiological and pathological conditions are poorly defined. To demonstrate a putative function of TGF- $\beta 1$ in the regulation of endometrial physiology, we performed an immunohistochemistry analysis of TGF- $\beta 1$ and its receptors $T \beta R I$ and $T \beta R I I$ on tissue sections of normal endometrium. Immunostaining of $T \beta R I$ and $T \beta R I I$ demonstrated that TGF- $\beta$ receptors were expressed in both endometrial epithelial and stromal cells. In contrast, TGF- $\beta 1$ expression was detected only in the stromal compartment (Supplementary Figure 1A). Next, we analyzed the response of normal endometrial cells to TGF- $\beta$ treatment. For this purpose, we established three-dimensional (3D) cultures of wild-type mouse endometrial epithelial cells. Under 3D culture conditions, endometrial epithelial cells develop polarized glandular structures that resemble in vivo uterine endometrial glands (Supplementary Figure 1B). These cultures provide a good scenario to study cell autonomous responses to TGF- $\beta{ }^{25}$ We confirmed that $T \beta R I$ and $T \beta R I I$ expression was retained in $3 D$ cultures of endometrial epithelial cells (Supplementary Figure 1C). Next, 3D cultures were exposed to TGF- $\beta$ for 12,24 or $36 \mathrm{~h}$. TGF- $\beta$ treatment caused a dose-dependent increase of nuclei displaying chromatin condensation and nuclear fragmentation typical of apoptotic cell death (Figures $1 \mathrm{a}$ and b). TGF- $\beta$-induced apoptosis was further analyzed by immunofluorescence analysis of cleaved caspase-3 (Figures $1 \mathrm{a}$ and b) and by western blot analysis of cleaved caspase-3 and -9 (Figure 1c). Progressive death of 3D glandular structures was also observed and monitored by time-lapse microscopy (Supplementary Movie 1, untreated gland and Supplementary Movie 2 TGF- $\beta$-treated gland). These results suggest that TGF- $\beta$ binding to its receptors triggers apoptotic cell death of polarized endometrial epithelial cells. To demonstrate that apoptosis was triggered by the activation of TGF- $\beta$ signaling, we treated $3 \mathrm{D}$ cultures with TGF- $\beta$ in presence or absence of the T $\beta \mathrm{RI}$ inhibitor, SB431542. Quantification of active caspase-3 immunofluorescence and nuclei displaying apoptotic morphology demonstrated that SB431542 was able to block TGF- $\beta$-induced apoptosis (Figure $1 \mathrm{~d}$ ). These results suggest that activation of TGF- $\beta$ signaling is required to induce apoptosis of endometrial epithelial cells.

To further investigate the molecular mechanism of TGF- $\beta$ pro-apoptotic function in polarized endometrial cells, we performed a RT-quantitative real-time PCR (qPCR) analysis of genes involved in apoptosis regulation on 3D cultures exposed to TGF- $\beta$ for $16 \mathrm{~h}$. Among all the pro-apoptotic members of the Bcl-2 family genes analyzed (Supplementary Figure 2), we found an increased expression of the proapoptotic BH3-only proteins BIM, BMF and NOXA (Figure 1e). Addition of the BH3-only mimetic ABT-263 to the 3D cultures increased the number of apoptotic cells to the same extent as TGF- $\beta$ treatment, suggesting that $\mathrm{BH} 3-$ only BIM, BMF or NOXA upregulation participates in TGF- $\beta$-induced apoptosis (Figure 1f).

Specific Smad3 deficiency hampers TGF- $\beta$-induced apoptosis. The role of $\mathrm{R}$-Smads in pro-apoptotic signaling triggered by TGF- $\beta$ is not completely known and both Smaddependent and Smad-independent mechanisms have been reported. ${ }^{11,26}$ For this reason, we analyzed $\mathrm{R}-S m a d$ nuclear translocation upon TGF- $\beta$ treatment. TGF- $\beta$ stimulation of 3D cultures resulted in a rapid nuclear translocation of Smad2/3 (Figure 2a). To ascertain the role of such translocation in apoptosis triggered by TGF- $\beta$, we infected wild-type endometrial cells with lentiviruses carrying shRNAs targeting Smad2 (FSV-Smad2.1 and FSV-Smad2.2) or Smad3 (FSVSmad3.1 and FSV-Smad3.2) (Supplementary Figure 3a).

Figure 1 TGF- $\beta$ triggers apoptosis of polarized endometrial epithelial cells. (a) Representative images of phase contrast (upper panel) and double tubulin and cleaved caspase-3 immunofluorescence (bottom) corresponding to endometrial 3D cultures treated with $10 \mathrm{ng} / \mathrm{ml}$ of TGF- $\beta$ for the indicated times. Nuclei were counterstained with Hoechst to show apoptotic nuclear morphology. Data are from $n=3$ experimental replicates (independent 3D cultures). Scale bars: $25 \mu$ m. (b) Quantification of nuclei displaying apoptotic morphology (upper graph) and cleaved caspase-3-positive immunostaining (bottom graph) in 3D cultures treated with the indicated doses of TGF- $\beta$ for the indicated times. Values are mean and error bars represent mean \pm S.E.M. ${ }^{\star} P<0.05$; ${ }^{\star *} P<0.01$ by one-way ANOVA, followed by the Tukey's multiple comparison test. (c) Western blot analysis of cleaved caspase- 3 and caspase- 9 from lysates of 3D cultures treated with $10 \mathrm{ng} / \mathrm{ml}$ of TGF- $\beta$ for the indicated times. Membrane was reblotted with tubulin to show equal protein loading. (d) Representative images (top) and quantification (bottom) of cells displaying apoptotic nuclear morphology and positive caspase-3 immunofluorescence corresponding to endometrial 3D cultures treated with $10 \mathrm{ng} / \mathrm{ml}$ of TGF- $\beta$ for $36 \mathrm{~h}$ in the presence or absence of SB431542. Nuclei were counterstained with Hoechst to show apoptotic nuclear morphology. Data are from $n=3$ experimental replicates (independent 3D cultures). Scale bar: $25 \mu \mathrm{m}$. (e) RT-qPCR analysis of BIM, BMF and NOXA mRNA expression in $3 \mathrm{D}$ cultures treated for $16 \mathrm{~h}$ with $10 \mathrm{ng} / \mathrm{ml}$ of TGF- $\beta$. Results are expressed as mRNA expression relative to untreated (UN) 3D culture condition. Values are mean \pm S.E.M. ${ }^{* *} P<0.01$, by $t$-test analysis. (f) Representative images of cleaved caspase-3, Hoechst and phalloidin staining (left), and quantification (right) of cleaved caspase3 cells in 3D cultures treated with the indicated doses of BH3 mimetic ABT-263 in combination or not (UN) with TGF- $\beta$ for 24 h. Scale bar: $25 \mu$ m. Graph values are the mean and error bars represent mean \pm S.E.M. ${ }^{\star \star} P<0.01$; NS (not significant) was calculated by one-way ANOVA analysis followed by Bonferroni's test 


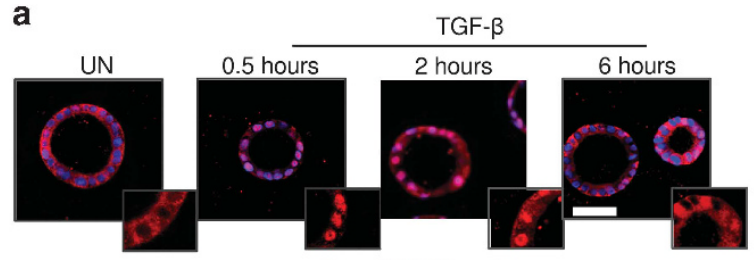

Smad 2/3 /Hoechst

b

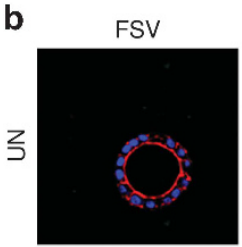

FSV-Smad 3.1

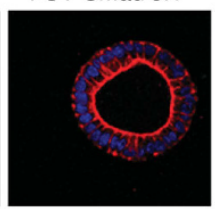

FSV-Smad 3.2

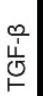
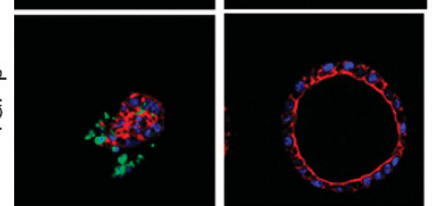

Cleaved Casp-3 / Tubulin / Hoechst

d
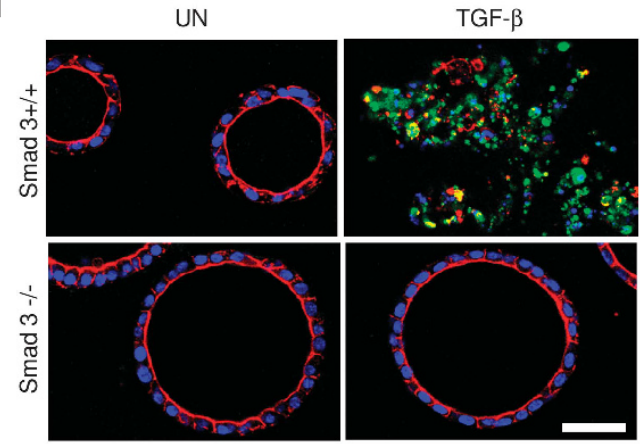

Cleaved-Casp3 / Phalloidin / Hoechst

f

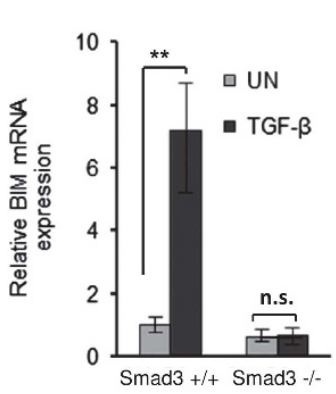

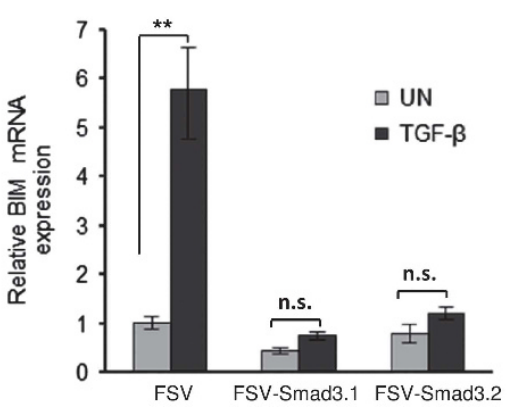

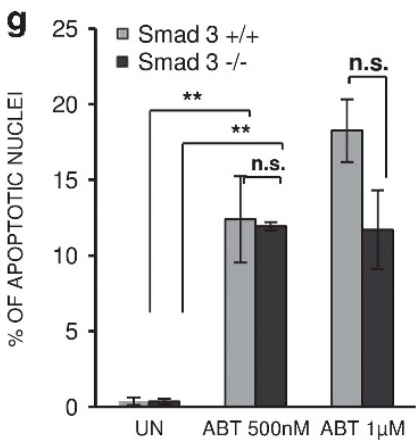

Figure 2 Smad3 deficiency results in resistance to TGF- $\beta$-induced (a) representative images of Smad2/3 immunofluorescence of 3D cultures untreated (UN) or treated with TGF- $\beta(10 \mathrm{ng} / \mathrm{ml})$. Nuclei were counterstained with Hoechst to show apoptotic nuclear morphology. Scale bar: $25 \mu \mathrm{m}$. (b) Representative cleaved caspase-3 immunostaining of endometrial 3D cultures infected with the lentiviruses carrying Smad3 shRNAs (FSV-shRNA3.1 or FSV-shRNA3.2) and UN or treated with 10 ng/ml of TGF- $\beta$ for 36 h. Cultures were immunostained with tubulin to show glandular structures. Nuclei were counterstained with Hoechst to show apoptotic nuclear morphology. Scale bar: $25 \mu$ m. (c) Quantification of cleaved caspase-3-positive cells in the same culture conditions. Values are mean \pm S.E.M. ${ }^{* *} P<0.01$, by $t$-test analysis. (d) Representative images and quantification of Smad3+/+ and Smad3 - / - endometrial 3D cultures UN or treated with $10 \mathrm{ng} / \mathrm{ml}$ of TGF- $\beta$ for $36 \mathrm{~h}$ and immunostained for cleaved caspase-3. Cultures were also immunostained with phalloidin to show glandular structures. Nuclei were counterstained with Hoechst to show apoptotic nuclear morphology. Scale bars: $25 \mu \mathrm{m}$. (e) Quantification of cleaved caspase-3-positive cells in the same culture conditions. Values are mean \pm S.E.M. ${ }^{* *} P<0.01$, by $t$-test analysis. (f) RT-qPCR analysis of BIM mRNA expression in 3D cultures from Smad3+/+ and Smad3-/ - or in 3D cultures infected with Smad3 shRNAs (FSV-Smad3.1 and FSV-Smad3.2) that were UN or treated with TGF- $\beta(10 \mathrm{ng} / \mathrm{ml})$ for $16 \mathrm{~h}$. Values are mean \pm S.E.M. ${ }^{* *} P<0.01$, by $t$-test analysis. $(\mathbf{g})$ Quantification of cleaved caspase-3-positive cells from Smad3+/+ and Smad3 - / - endometrial 3D cultures UN or treated with ABT-263. Values are mean and error bars represent mean \pm S.E.M. ${ }^{* \star} P<0.01$ by one-way ANOVA, followed by the Tukey's multiple comparison test 
Eight to 10 days after infection, 3D cultures were exposed to TGF- $\beta$. Interestingly, Smad3 shRNAs (Figure 2b) caused a decrease of cells displaying positive immunostaining for cleaved caspase-3 after TGF- $\beta$ treatment. Quantification of the number of apoptotic cells per gland revealed that shRNAinduced downregulation of Smad3 resulted in an almost complete blockade of apoptosis triggered by TGF- $\beta$ (Figure 2c). To rule out putative unspecific effects of shRNA, we established 3D endometrial cultures from Smad3 knockout mice. Smad3 knockout blocked the increase of the number of cells displaying positive immunofluorescence for cleaved caspase-3 after TGF- $\beta$ treatment (Figures $2 d$ and e). Conversely, either Smad2 downregulation or Smad2 knockout had no effects on the number of apoptotic cells after TGF- $\beta$ treatment (Supplementary Figure 3B). Consistently, either Smad3 knockout or knockdown prevented TGF- $\beta$-induced upregulation of BIM mRNA expression (Figure 2f). Noteworthy, treatment of Smad3-deficient cells with the BH3-only mimetic ABT-263 triggered the same levels of apoptosis as in wild-type cells (Figure $2 \mathrm{~g}$ ).

Specific ablation of Smad3 enhances epithelial cell proliferation. Besides the inhibition of TGF- $\beta$-induced apoptosis, observation of Smad3 - / - or Smad3 shRNA-infected 3D cultures evidenced a marked increase of glandular size over their wild-type counterparts (Figure 3a). Measurements of glandular perimeter confirmed an increase of glandular size in either 3D cultures transduced with Smad3 shRNAs (Figure 3a) or 3D cultures from Smad3 knockout mice (Figure 3b), suggesting that Smad3 deficiency enhances endometrial epithelial cell proliferation. To demonstrate such hypothesis, we measured the levels of cyclin D1 mRNA expression and we performed BrdU incorporation assays. Smad3-/- 3D cultures or cultures infected with Smad3 shRNA displayed a significant increase of cyclin D1 expression (Figure 3c) and the number of BrdU-incorporating cells (Figure 3d). In contrast, Smad2 downregulation had no effects on cyclin D1 expression (Supplementary Figure $3 \mathrm{C}$ ), the number of BrdU-incorporating cells (Supplementary Figure 3D) and glandular size (Supplementary Figure 3E). Consistently, Smad2-/- cells did not display increased glandular size (Supplementary Figure $3 E$ ). These results strongly support the hypothesis that Smad3 deficiency enhances cell proliferation of polarized endometrial epithelial cells.

Smad3 regulates PTEN protein levels and AKT activation. Alterations of $\mathrm{PI} 3 \mathrm{~K} / \mathrm{AKT}$ signaling pathway are frequently found in endometrial cancer and other malignancies. ${ }^{13}$ Therefore, we sought to investigate whether TGF- $\beta /$ Smad3 signaling could be regulating the activation of the PI3K/AKT signaling pathway. To test such hypothesis, we performed a western blot analysis of different components of the PI3K/ AKT signaling pathway on 3D cultures infected with lentiviruses carrying Smad3 shRNAs (Figure 4a) or on 3D cultures established from Smad3 knockout mice (Figure 4b). Both knockdown or knockout Smad3 cells displayed a reduction of PTEN expression and an increase of AKT phosphorylation (Figures $4 a$ and b). Furthermore, the downstream targets of $\mathrm{PI} / \mathrm{K} / \mathrm{AKT} / \mathrm{mTOR}$ signaling pathway p70S6K and eiF4E displayed higher phosphorylation levels in Smad3-deficient cells than in wild-type cells. Consistently, TGF- $\beta$ treatment of 3D cultures caused a significant increase of PTEN protein levels and AKT phosphorylation (Figure 4c). Conversely, neither Smad2 knockdown nor Smad2 knockout modified the levels of PTEN protein expression (Supplementary Figures 4A, 4B).

Next, we investigated whether the Smad3-dependent regulation of PTEN expression takes place in vivo. To address this question, we carried out PTEN and p-AKT immunohistochemistry on uterine tissue sections from Smad3+/+ and Smad3-/ - littermates. Serial sections from four different Smad3 - / - littermates mice uterus displayed a significant reduction of $\mathrm{PTEN}$ immunostaining and an increased staining for $\mathrm{p}$-AKT compared to the uterus of their wild-type littermates (Figure 4d; Table 1).

Smad3 binds PTEN promoter to enhance its transcription. Next, we investigated the molecular mechanism by which Smad3 regulates PTEN protein levels. We first questioned whether regulation of PTEN protein levels could be caused by a Smad3-dependent regulation of PTEN transcription. For this purpose, we measured PTEN mRNA levels by RT-qPCR on Smad3-deficient cells. ShRNAmediated downregulation of Smad3 in 3D cultures resulted in a reduction of PTEN mRNA levels (Figure 5a). Analysis of four different Smad3 knockout litters showed a dramatic reduction of PTEN mRNA levels in Smad3-/- epithelial endometrial cells compared to their wild-type littermates (Figure 5b). Importantly, Smad2 downregulation had no effect on PTEN expression (Supplementary Figure 5A).

Next, we analyzed the role of Smad3 in TGF- $\beta$-induced PTEN expression. For this purpose, we measured PTEN mRNA levels in wild-type 3D cultures treated for different periods of time with TGF- $\beta$ or TGF- $\beta$ plus the T $\beta$ RI inhibitor SB431542. RT-qPCR analysis revealed a significant increase of PTEN mRNA in response to TGF- $\beta$, which was completely abrogated by SB431542 (Figure $5 \mathrm{c}$ ). Consistently, treatment of 3D cultures with SB431542 effectively blocked TGF- $\beta$ induced nuclear translocation of Smad2/3 (Supplementary Figure 5B). Moreover, TGF- $\beta$-induced increase of PTEN mRNA was prevented by the transcription inhibitor actinomycin D, but not by the translation inhibitor cycloheximide (Supplementary Figure 5C), further supporting that TGF- $\beta$ / Smad3-signaling regulates PTEN transcription. Finally, we measured PTEN mRNA levels in Smad3-deficient cultures treated with TGF- $\beta$ for $12 \mathrm{~h}$. Smad3 - / - cultures displayed an almost total inhibition of TGF- $\beta$-induced PTEN mRNA expression (Figure $5 \mathrm{~d}$ ). The apoptosis triggered by TGF- $\beta$ in wild-type cells prevented the analysis of PTEN expression at longer time points. However, 3D cultures infected with Smad3 shRNAs or knockout from Smad3, the decrease in PTEN expression was maintained even $96 \mathrm{~h}$ after TGF- $\beta$ treatment (Supplementary Figure 5D).

To provide direct evidence that PTEN is a target gene of Smad3 transcriptional activity, we performed a chromatin immunoprecipitation (ChIP) analysis of PTEN promoter using Smad2- or Smad3-specific antibodies on unstimulated or TGF- $\beta$-treated 3D cultures. To identify putative Smad-binding sites on PTEN promoter, we analyzed three sequential $500 \mathrm{bp}$ segments spanning from position -1 to -1500 of PTEN 

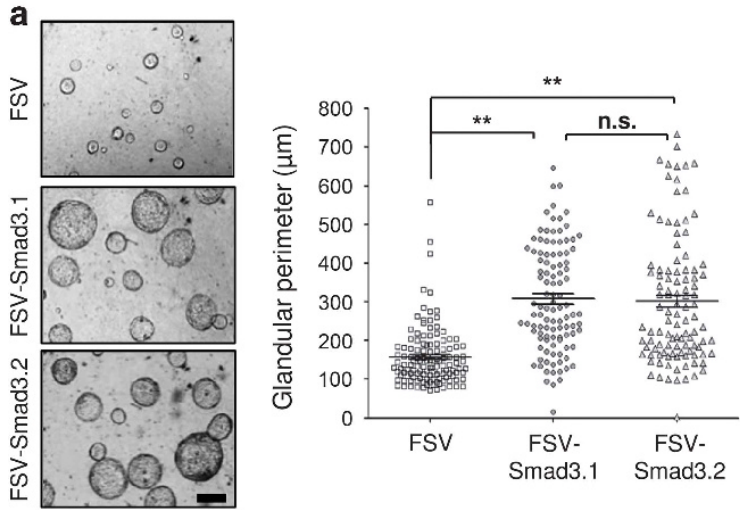

b
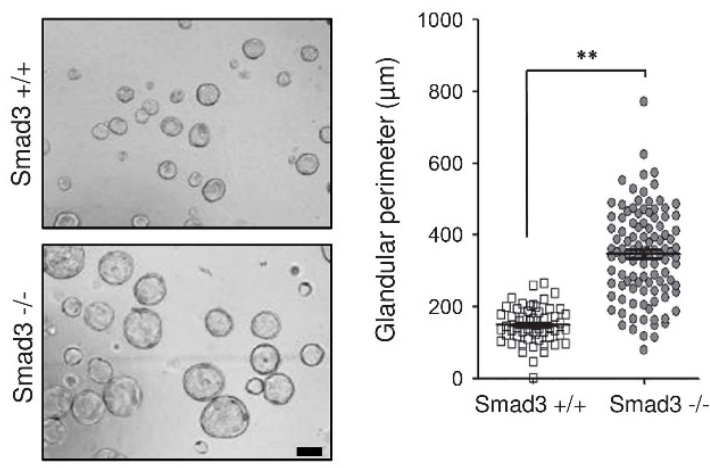

C
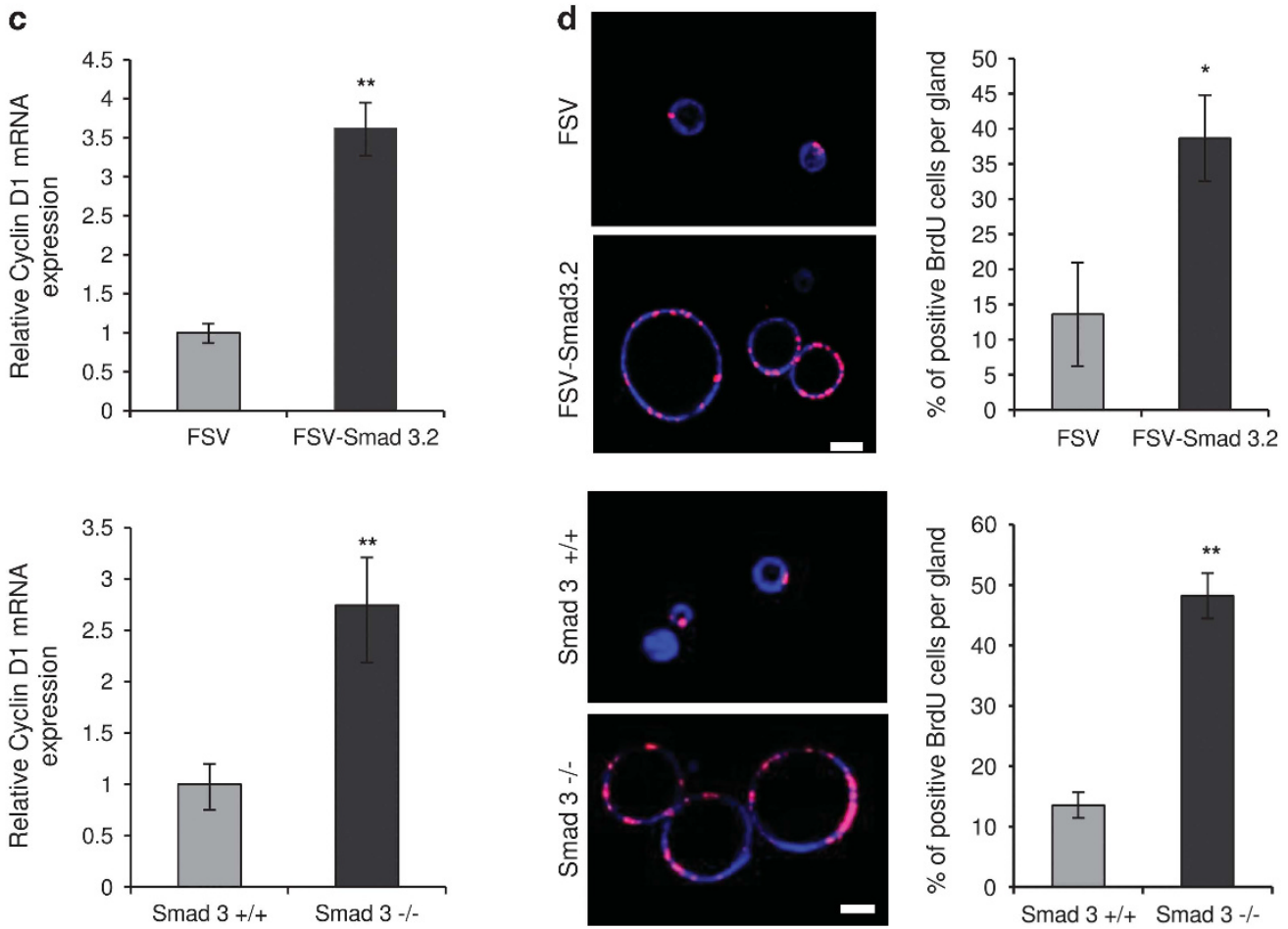

Figure 3 Smad3 deficiency increases cell proliferation. Representative phase contrast images and measurement of gland perimeter corresponding to: (a) 3D cultures infected with Smad3 shRNAs (FSV-Smad3.1 and FSV-Smad3.2) or (b) 3D cultures from Smad3+/+ and Smad3 - / - cells. Errors bars represent mean \pm S.E.M. ${ }^{* *} P<0.01$ by t-test analysis. Scale bars: $50 \mu \mathrm{m}$. (c) RT-qPCR analysis of cyclin D1 mRNA expression from 3D cultures infected with Smad3 shRNA (FSV-Smad3.2) or 3D cultures from Smad3 $+/+$ and Smad3 $-/$ - cells. Values are mean \pm S.E.M. ${ }^{* *} P<0.01$, by $t$-test analysis. (d) Upper panel, representative images and quantification of BrdU-positive cells in $3 D$ cultures infected with Smad3 shRNA (FSV-Smad3.2). Lower panel, representative images and quantification of BrdU-positive cells from Smad3+/+ and Smad3 - / - 3D cultures. Nuclei were evidenced by Hoechst staining. Scale bars $=25 \mu \mathrm{m}$ Values are mean \pm S.E.M. ${ }^{* *} P<0.01,{ }^{*} P<0.05$, by $t$-test analysis. NS, not significant

promoter. TGF- $\beta$ treatment caused a dramatic increase of Smad3-dependent immunoprecipitation of the three PTEN promoter segments, suggesting that TGF- $\beta$ induces Smad3 binding to PTEN promoter (Figure 5e). Noteworthy, unstimulated samples displayed a basal binding of Smad3 to PTEN promoter. Conversely, there was no increase of any of the three PTEN promoter regions when ChIP analysis was performed using Smad2-specific antibody (Figure 5e). These results strongly suggest that Smad3, but not Smad2, binds to PTEN promoter to enhance its transcription upon TGF- $\beta$ stimulation.

The in vitro and in vivo results supporting a Smad3dependent regulation of PTEN protein levels in the endometrium encouraged us to investigate whether such mechanism could take place in other cells or tissues. For this reason, we analyzed PTEN mRNA expression in heart, spleen, thyroid and liver collected from Smad3 wild-type and knockout littermates. Interestingly, Smad3-/ - spleen and thyroid, but not heart and liver, displayed a significant reduction of PTEN expression when compared with their Smad3+/+ littermates (Supplementary Figure 5E). To examine TGF- $\beta$-induced upregulation of PTEN in another cell system, we treated 3D cultures of human HEK-293T cells with TGF- $\beta$ and we measured PTEN mRNA expression. Likewise in 3D cultures from endometrial murine cells, TGF- $\beta$ treatment increased PTEN mRNA expression of HEK-293T cells 

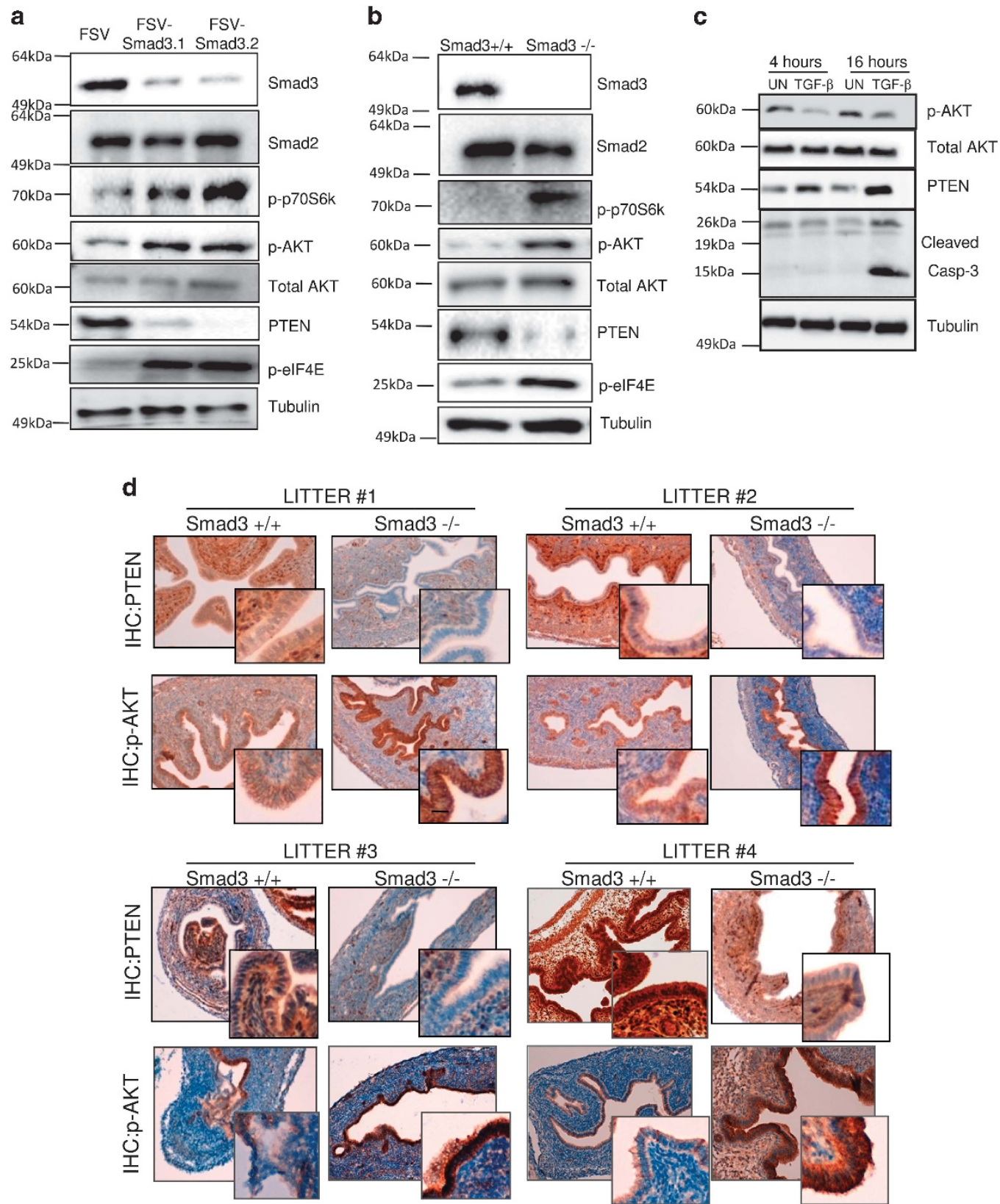

Figure 4 Smad3 regulates PTEN protein expression and AKT phosphorylation. (a) Western blot analysis of 3D cultures infected with Smad3 shRNAs (FSV-Smad3.1 and FSV-Smad3.2) displaying a decreased PTEN protein levels and an increased phosphorylation of AKT, p70S6k and eiF4E compared to controls (FSV). Membranes were reblotted with total AKT and tubulin to show equal protein loading. A representative image is shown. (b) Western blot analysis showing a decreased expression and increased phosphorylation of AKT, p70S6K and eiF4E in Smad3 - / - cells compared to Smad3+/+ cells. Membranes were reblotted with total AKT and tubulin to show equal protein loading. A representative western blot image is shown. (c) Western blot analysis of phoshorylated AKT, PTEN expression and cleaved caspase-3 in cell lysates from untreated cultures (UN) or cultures treated for 4 or $16 \mathrm{~h}$ with TGF- $\beta(10 \mathrm{ng} / \mathrm{ml})$. A representative image is shown. (d) Representative images of PTEN and phosphorylated AKT immunohistochemistry performed on serial endometrial tissue sections from four different Smad3+/+ and Smad3 - / - 4-week-old littermates (x20 images and magnification; $n=6 \mathrm{Smad} 3+/+$ and $n=4 \mathrm{Smad} 3-/$ - littermates)

(Supplementary Figure 5F). All these results suggest that Smad3-induced PTEN expression may be a molecular mechanism that operates in different tissues or organs.

Smad3-dependent PTEN expression regulates cell proliferation and TGF- $\beta$-induced apoptosis by a PI3K/ AKT-dependent mechanism. The above results led us to hypothesize that PTEN upregulation could be regulating cell proliferation and sensitivity to TGF- $\beta$-induced apoptosis downstream of Smad3 activation. To test such hypothesis, endometrial cells were infected with Smad3 shRNAs and treated with the PI3K inhibitor, LY294002. Addition of LY294002 to cell cultures infected with Smad3 shRNAs prevented the increase of glandular perimeter (Figure 6a). Next, we analyzed the effects of PI3K inhibition on TGF- $\beta$-induced apoptosis. Treatment of 3D cultures infected with Smad3 shRNAs (Figure 6b) 
or 3D cultures from Smad3 - / - mice (Figure 6c) with TGF- $\beta$ plus LY294002 resulted in a marked increase of cells displaying positive immunostaining for cleaved caspase-3 and cells displaying apoptotic morphology. These results suggest that enhanced PI3K/AKT signaling caused by Smad3 deficiency inhibits pro-apoptotic function of TGF- $\beta$.

PTEN deficiency enhances cell proliferation and TGF- $\beta$-induced apoptosis by a PI3K/AKT-dependent mechanism. To provide a direct evidence that activation of PI3K/AKT signaling pathway caused by PTEN loss was important to regulate epithelial cell proliferation and pro-apoptotic function of TGF- $\beta$, we analyzed the effects of TGF- $\beta$ on PTEN-deficient endometrial epithelial cells. For this purpose, we established $3 D$ endometrial cultures from mice carrying both PTEN-floxed alleles (Pten fl/fl) and the tamoxifen-inducible Cre recombinase (Cre:ER+/-). Addition of tamoxifen to endometrial epithelial cells isolated from Cre: $\mathrm{ER}+\mathrm{l}-$; Pten $\mathrm{fl} / \mathrm{fl}$ mice resulted in an efficient PTEN deletion and concomitant increase in AKT phosphorylation (Supplementary Figures 6A and B). TGF- $\beta$ treatment of PTEN knockout 3D cultures resulted in an almost complete suppression of nuclei displaying nuclear apoptotic morphology and the number of cells displaying positive staining for cleaved caspase-3, even after $72 \mathrm{~h}$ of TGF- $\beta$ treatment (Figures $7 \mathrm{a}$ and

Table $1 \mathrm{IHC}$ quantification results of PTEN and p-AKT by histological score evaluated by pathologists and by automated ASICS III system

\begin{tabular}{|c|c|c|c|c|c|}
\hline & \multicolumn{5}{|c|}{ PTEN immunostaining } \\
\hline & Min & $\operatorname{Max}$ & Mean & S.E.M. & $P$-value \\
\hline \multicolumn{6}{|l|}{ Pathologists } \\
\hline SMAD3+/+ & 60 & 290 & 125 & 45.55 & $0.0179^{*}$ \\
\hline SMAD3 - /- & 10 & 60 & 28.0 & 11.14 & \\
\hline \multicolumn{6}{|c|}{ ACIS III quantification } \\
\hline SMAD3+/+ & 108 & 145 & 115.5 & 5.334 & $0.0009^{*}$ \\
\hline \multirow[t]{2}{*}{ SMAD3 - /- } & 58 & 82 & 71.3 & 3.088 & \\
\hline & \multicolumn{5}{|c|}{ p-Akt immunostaining } \\
\hline \multicolumn{6}{|l|}{ Pathologists } \\
\hline SMAD3+/+ & 150 & 180 & 162.5 & 7.5 & 0.1734 \\
\hline SMAD3 - /- & 170 & 220 & 190 & 11.99 & \\
\hline \multicolumn{6}{|c|}{ ACIS III Quantification } \\
\hline SMAD3+/+ & 97 & 121 & 116.2 & 2.976 & $0.0289^{*}$ \\
\hline SMAD3 - /- & 125 & 157 & 137.5 & 4.164 & \\
\hline
\end{tabular}

Unpaired Student's $t$-test was assessed considering ${ }^{*} P<0.05$ b). Consistently, PTEN deficiency also caused a marked reduction of both basal and TGF- $\beta$-induced expression of the pro-apoptotic member of Bcl-2 family BIM (Figure 7c).

Finally, we also evaluated the effects of PTEN deficiency on cell proliferation. PTEN-deficient 3D cultures displayed an increased glandular perimeter (Figure 7d), which correlated with a significant increase of BrdU-incorporating cells (Figure 7e) and cyclin D1 mRNA expression (Figure 7f). All the above results demonstrate that PTEN loss regulates endometrial epithelial cell proliferation and the sensitivity to TGF- $\beta$-induced apoptosis.

Inhibition of PI3K/AKT/mTORC1 signaling reduces cell proliferation and restores TGF- $\beta$-induced apoptosis in PTEN-deficient cells. Finally, we studied whether enhanced PI3K/AKT activity was responsible for the increase of cell proliferation and the suppression of TGF- $\beta$-induced apoptosis in PTEN-deficient cells. PTEN-deficient 3D cultures were infected with lentiviruses carrying PTEN cDNA to restore PTEN expression and to decrease AKT activation. Cells overexpressing exogenous PTEN displayed a significant increase of caspase-3 activation and apoptotic nuclei upon TGF- $\beta$ treatment (Figure 8a). PTEN overexpression caused an expected reduction of AKT phosphorylation (Supplementary Figure $6 \mathrm{C}$ ). To further evidence the role of PI3K activation in blockade of TGF- $\beta$-induced apoptosis, PTEN-deficient 3D cultures were treated with TGF- $\beta$ plus LY294002. Inhibition of PI3K restored apoptosis triggered by TGF- $\beta$ (Figure 8b). Consistently, inhibition of PI3K also resulted in a significant reduction of glandular perimeter of PTEN-deficient cells (Figure $8 \mathrm{c}$ ). We further analyzed the role of $\mathrm{mTORC} 1$ in the transduction of proliferative and survival signals downstream of PI3K/AKT activation. Inhibition of mTORC1 with either Rapamycin or Everolimus caused a marked decrease of glandular perimeter of PTEN-deficient glands (Figure 8d). Everolimus and Rapamycin reduced the number of BrdU-positive cells in PTEN-deficient glands (Figure 8e). Importantly, Everolimus and Rapamycin also restored TGF- $\beta$-induced apoptosis of PTEN-deficient cells (Figure 8f). All these results support the hypothesis that mTORC1 is the molecular effector of Smad3 nuclear translocation in PTEN-deficient cells.

\section{Discussion}

The paradox of TGF- $\beta$ actions during carcinogenesis is still poorly understood. In the present work, we have unveiled a

\footnotetext{
Figure 5 Smad3 binds PTEN promoter to enhance its transcription. (a) RT-qPCR of PTEN mRNA expression in 3D cultures infected with Smad3 shRNAs (FSV-Smad3.1 and FSV-Smad3.2). Data are from $n=3$ experimental replicates and values are mean \pm S.E.M. ${ }^{*} P<0.01$, by one-way ANOVA post hoc Tukey's multiple comparison test. (b) RTqPCR analysis of PTEN mRNA expression of 3D cultures from four different Smad3+/+ and Smad3 - / - 4-week-old littermates. Values are mean \pm S.E.M. ${ }^{*} P<0.05$ and ${ }^{*} P<0.01$, by t-test analysis. (c) RT-qPCR analysis showing PTEN mRNA expression in 3D cultures treated for the indicated times with $10 \mathrm{ng} / \mathrm{ml}$ of TGF- $\beta$ alone or TGF- $\beta$ plus SB431542. Values are mean \pm S.E.M. ${ }^{*} P<0.01$, by $t$-test analysis. (d) RT-qPCR analysis of PTEN mRNA in 3D cultures from Smad3+/+ and Smad3 $-/-$ epithelial cells treated for $12 \mathrm{~h}$ with $10 \mathrm{ng} / \mathrm{ml}$ of TGF- $\beta$ or untreated (UN). Values are mean \pm S.E.M. ${ }^{* *} P<0.01$, by $t$-test analysis. (e) ChIP analysis of Smad2/3 binding to PTEN promoter. Cell lysates from UN or TGF- $\beta$-stimulated 3D cultures (30 min) were subjected to immunoprecipitation (IP) with Smad2, Smad3 or control antibody (IgG). Immunoprecipitates were analyzed by real-time qPCR using primers to amplify three $~ 500$ bp consecutive segments of PTEN promoter $(-1 /-500,-501 /-986,-987 /-1459)$. Plots show the results from qPCR analysis of PTEN promoter regions expressed as fold enrichment of PTEN promoter amplification over the input DNA. Results correspond to $n=3$ experimental replicates and values are mean \pm S.E.M. Right panel shows a representative gel image of ChIP analysis (conventional electrophoresis gel run of the samples amplified in real-time experiments)
} 
novel regulatory loop between TGF- $\beta /$ Smad3 and PI3K/AKT signaling pathways that regulates proliferation and apoptotic cell death.

TGF- $\beta$ is important for the regulation of endometrial development and function. ${ }^{20}$ Here, we have found that TGF- $\beta$ is only expressed in stromal cells, while $T \beta R I$ and $T \beta R$ II are expressed in both stromal and epithelial compartments suggesting that TGF- $\beta$ is produced by stromal cells and acts in a paracrine way on epithelial cells to regulate their functions. We have demonstrated that TGF- $\beta$ induces apoptosis of

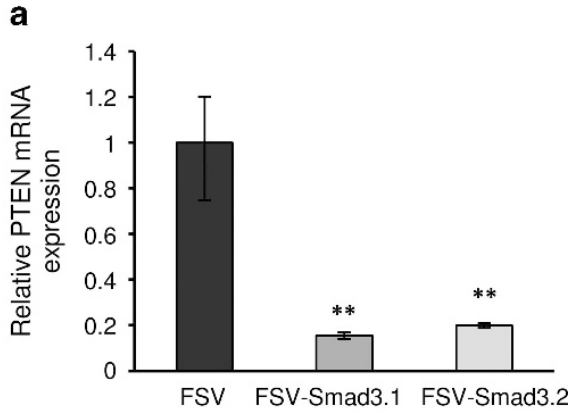

c

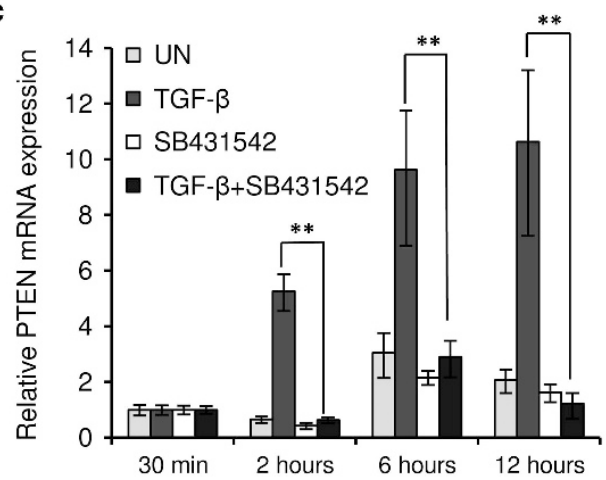

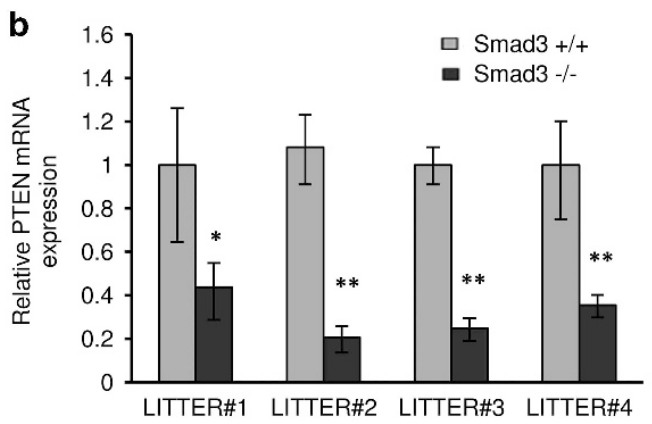

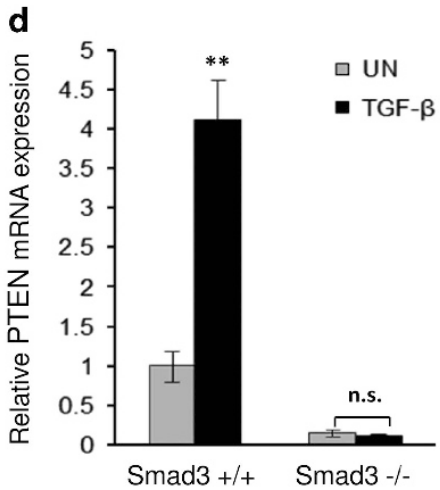

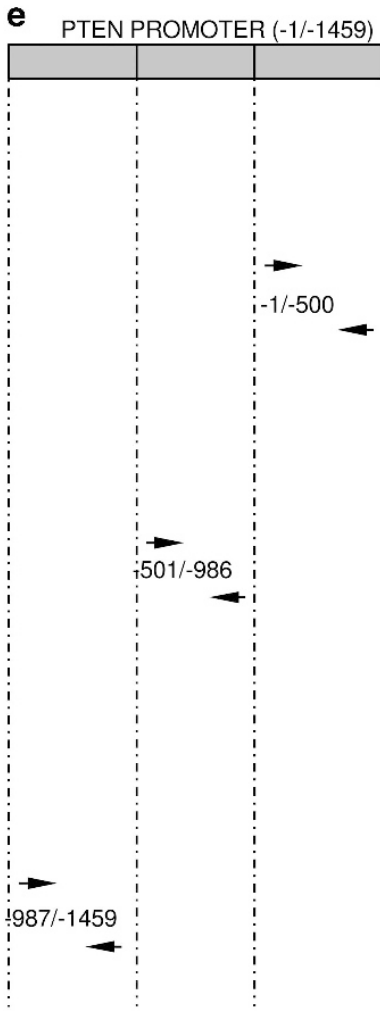
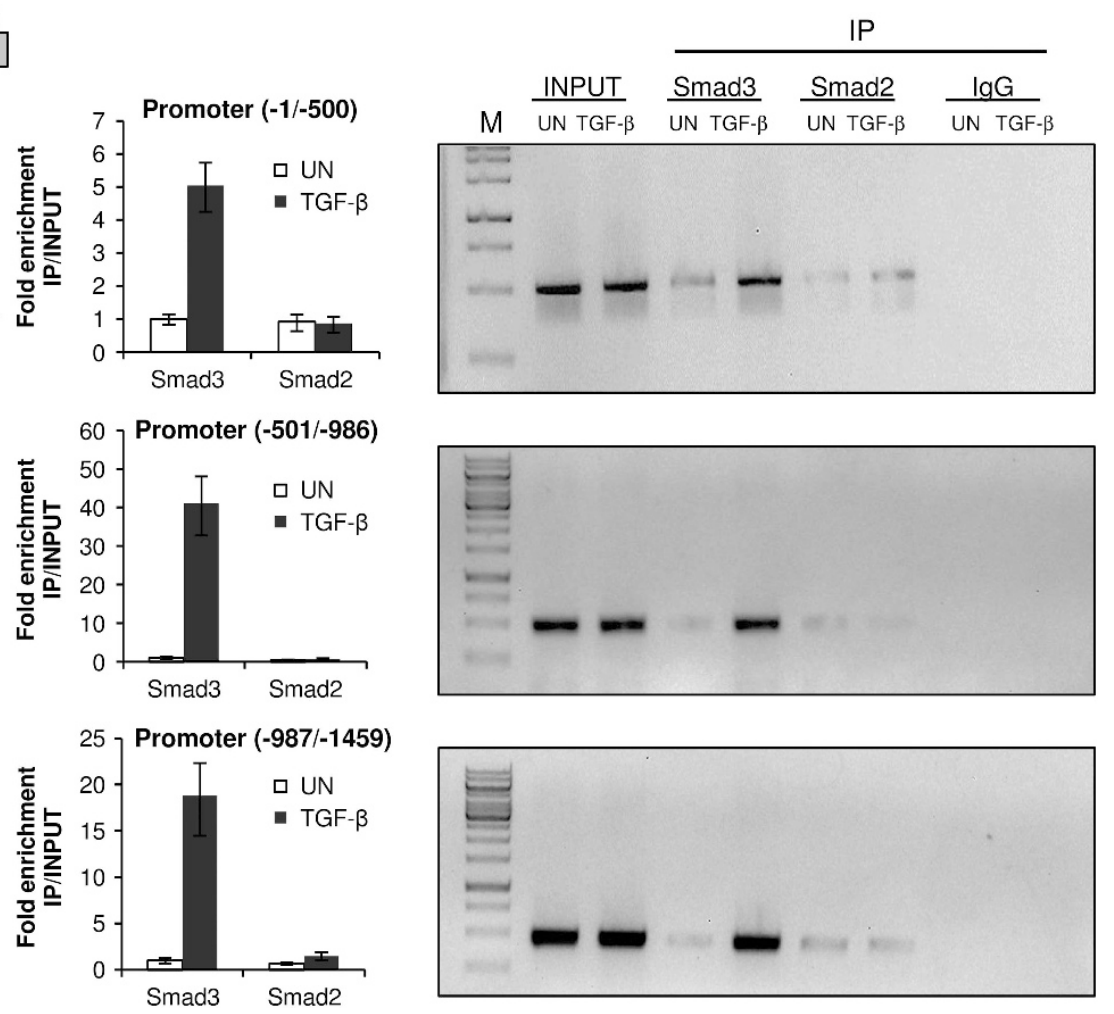
a

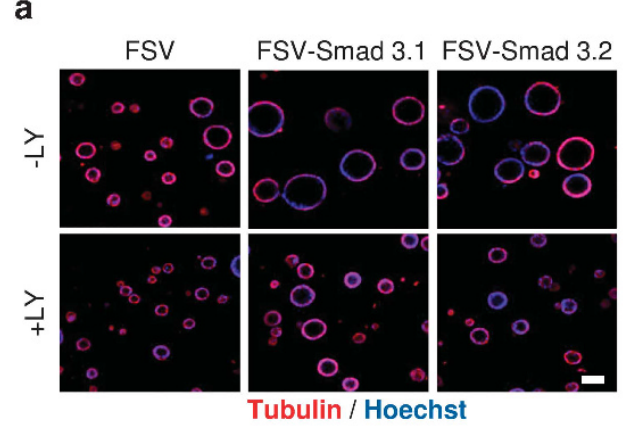

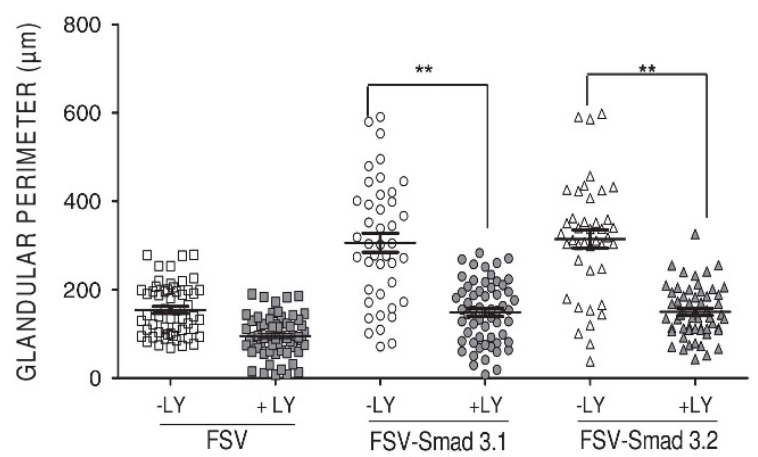

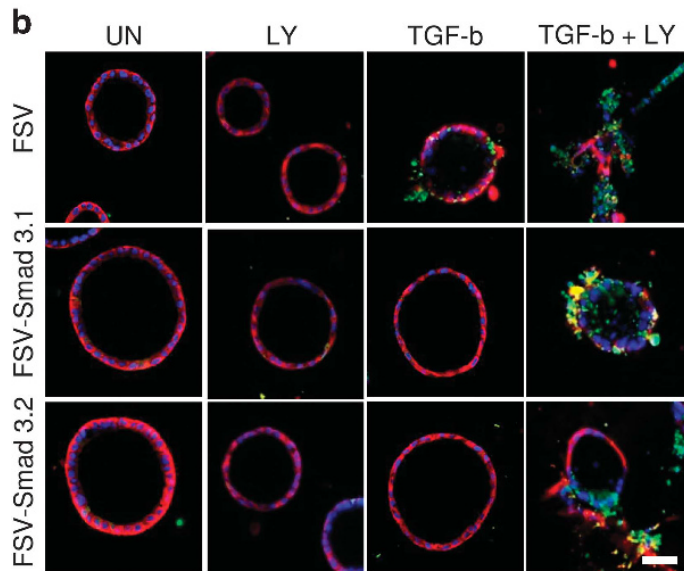

Cleaved Caspase-3 / Tubulin / Hoechst

c

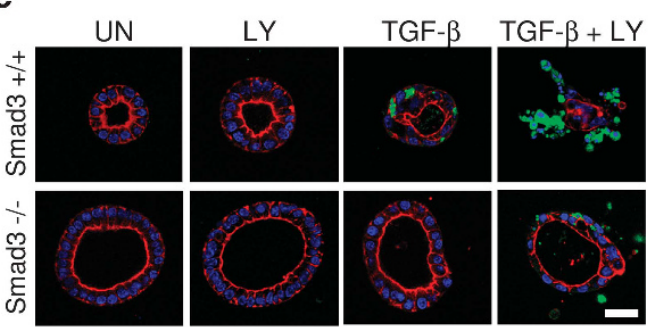

Cleaved Caspase-3 / Tubulin / Hoechst
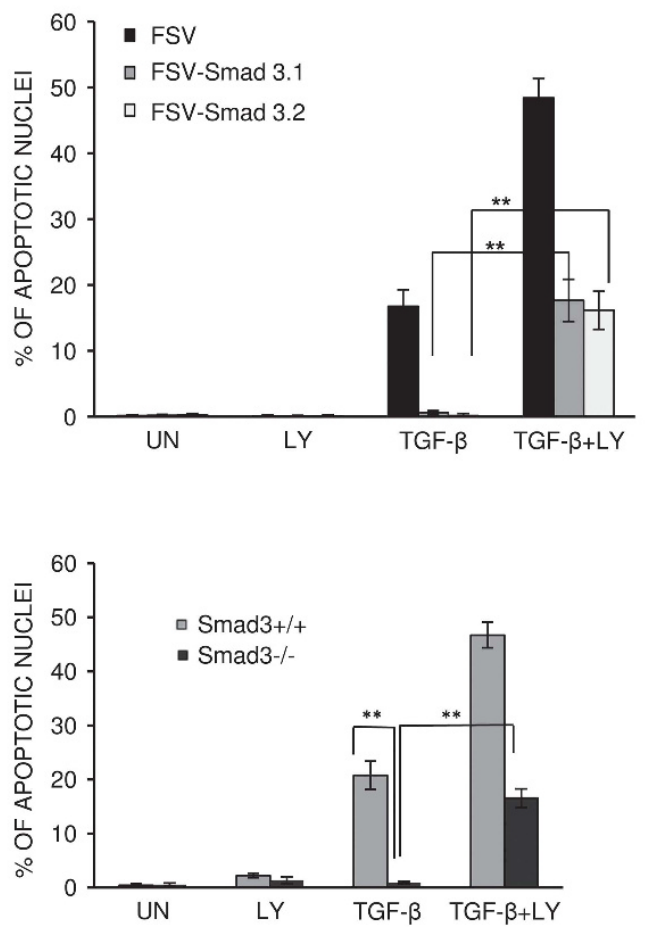

Figure 6 PI3K/AKT inhibition and PTEN expression regulate TGF- $\beta$-induced apoptosis. (a) 3D cultures infected with Smad3 shRNAs (FSV-Smad3.1 and FSV-Smad3.2) and treated $(+\mathrm{LY})$ or not $(-\mathrm{LY})$ with $10 \mu \mathrm{M}$ of $\mathrm{LY} 294002$. Left panel shows representative images of 3D cultures stained with tubulin antibodies and Hoechst to show glandular structures under the indicated conditions. Right plot shows a quantification of glandular perimeter. Errors bars represent mean \pm S.E.M. ${ }^{*} P<0.01$ by $t$-test analysis. Scale bar: $50 \mu \mathrm{m}$. (b) Left panel, representative images corresponding to cleaved caspase-3/tubulin/Hoechst staining on 3D cultures infected with Smad3 shRNAs (FSV-Smad 3.1 and FSVSmad 3.2) or the control vector (FSV) and stimulated with TGF- $\beta(10 \mathrm{ng} / \mathrm{ml}$ ) alone or in combination with the PI3K inhibitor LY294002 (LY). Data are from $n=3$ experimental replicates. Scale bar: $25 \mu \mathrm{m}$. Right graph, quantification of positive staining for cleaved caspase-3 in cells exposed to the treatment described. Values are mean \pm S.E.M. ${ }^{* *} P<0.01$, by $t$-test analysis. (c) Representative pictures of cleaved caspase-3, Hoechst and tubulin staining (left panel) and quantification (right graph) of cleaved caspase-3positive cells from Smad3+/+ and Smad3 - / - cultures pre-treated with $10 \mu \mathrm{M}$ of LY294002 (+LY) for $2 \mathrm{~h}$ in combination with no further treatment (UN) or with TGF- $\beta$ (10 ng/ml) treatment for $24 \mathrm{~h}$. Scale bar: $25 \mu \mathrm{m}$. Values are mean and error bars represent mean \pm S.E.M. ${ }^{* *} P<0.01$ by two-way ANOVA followed by Bonferroni post hoc analysis

normal polarized endometrial cells. To date, the molecular mechanisms by which TGF- $\beta$ triggers apoptosis are not fully understood. ${ }^{26,27}$ Previous studies have shown that upregulation of BH3-only proteins is important for TGF- $\beta$-induced apoptosis. ${ }^{28,29}$ Among them, BIM has been reported to be essential for TGF- $\beta$ apoptosis. ${ }^{30-32}$ We have shown that TGF- $\beta$ induces the expression of the $\mathrm{BH} 3$-only proteins BIM, NOXA and BMF in a Smad3-dependent manner. The blockade of TGF- $\beta$-induced apoptosis caused by ablation of either Smad3 or PTEN correlates with the inhibition of TGF- $\beta$ induced upregulation of BIM. These results support a role for the $\mathrm{BH} 3$-only proteins in the regulation of pro-apoptotic TGF- $\beta$ signaling on endometrial epithelial cells.

Previous studies suggested that TGF- $\beta$ may participate in endometrial cancer progression. It has been reported that TGF- $\beta$ signaling is disrupted in endometrial carcinogenesis ${ }^{33}$ and, downregulation of TGF- $\beta$ signaling has been associated to poor prognosis. ${ }^{34}$ Moreover, in early endometrial 
a

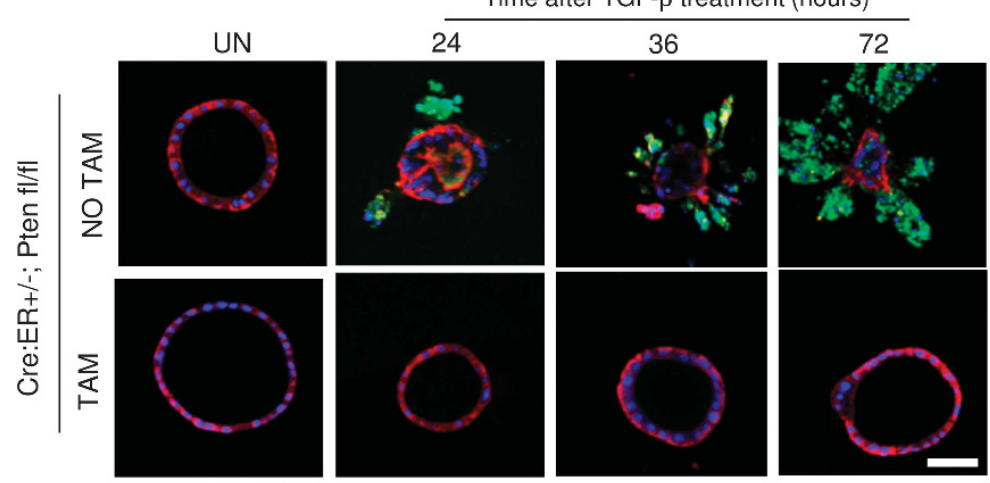

Cleaved-Casp-3/Tubulin/Hoechst
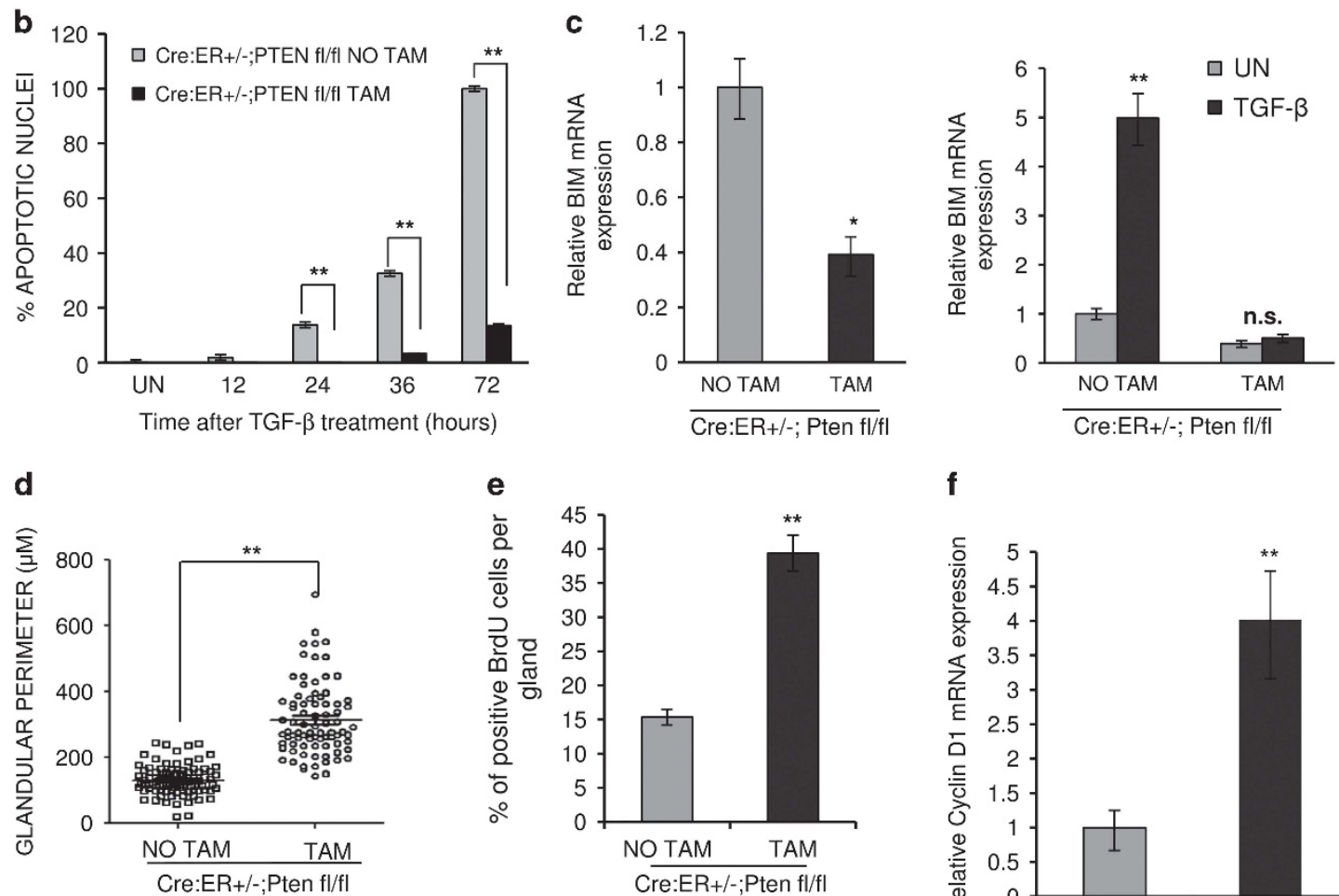

f

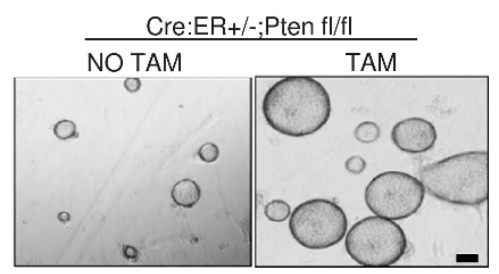

Cre:ER+/-;Pten fl/fl
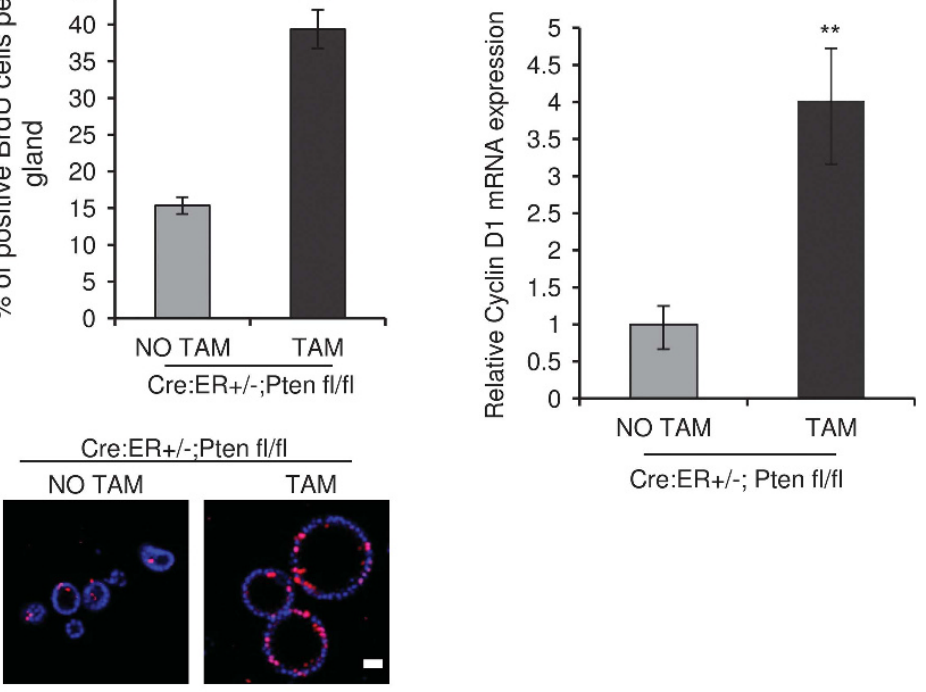

Cre:ER+/-; Pten fl/fl

Figure 7 PTEN deficiency increases cell proliferation and impairs TGF- $\beta$-induced apoptosis. (a) Representative images of cleaved caspase-3 immunostaining performed on 3D cultures from Cre:ER+/ - ; Pten fl/fl exposed (TAM) or not (NO TAM) to tamoxifen to induce PTEN deletion; and treated or not (UN) with TGF- $\beta$ (10 ng/ml) for the indicated times. 3D cultures were also stained with tubulin and Hoechst to show glandular structures and nuclear morphology. Scale bars: $25 \mu \mathrm{m}$. (b) Quantification of the cells displaying positive cleaved caspase-3 immunostaining under the same conditions. Data are shown for five independent experiments (each with $n=180$ counted cells per condition). Values are mean \pm S.E.M. ${ }^{* *} P<0.01$, by $t$-test analysis. (c) Left graph shows a RT-qPCR analysis of BIM expression of 3D cultures from Cre:ER+/ - ; Pten fl/fl 3D cultures treated (TAM) or not (NO TAM) with tamoxifen to induce PTEN deletion. Right graph shows a RT-qPCR analysis of BIM expression of 3D cultures from Cre:ER+/ - ; Pten fl/fl 3D cultures treated (TAM) or not (NO TAM) with tamoxifen and then untreated (UN) or treated with $10 \mathrm{ng} / \mathrm{ml}$ of TGF- $\beta$ for $16 \mathrm{~h}$. Values are mean \pm S.E.M. ${ }^{*} P<0.05$ and ${ }^{* *} P<0.01$, by $t$-test analysis. (d) Measurement of glandular perimeter and representative phase contrast images of $3 \mathrm{D}$ cultures from Cre:ER+l - ; Pten fl/fl $3 \mathrm{D}$ cultures treated (TAM) or not (NO TAM) with tamoxifen to induce PTEN deletion. All the experiments were performed at least three times. Error bars represent mean \pm S.E.M. ${ }^{\star \star} P<0.01$ by t-test analysis. Scale bars: $25 \mu$ m. (e) Quantification and representative images of BrdU-positive cells in 3D cultures from Cre:ER+/ - ; Pten fl/fl 3D cultures treated (TAM) or not (NO TAM) with tamoxifen to induce PTEN deletion. Nuclei were evidenced by Hoechst staining. Scale bar $=25 \mu \mathrm{m}$ Data are from $n=3$ experimental replicates and values are mean \pm S.E.M. ${ }^{* *} P<0.01$, by $t$-test analysis. (f) $R T$-qPCR analysis of cyclin D1 mRNA expression on $3 \mathrm{D}$ cultures from Cre:ER+/ - ; Pten fl/fl 3D cultures treated (TAM) or not (NO TAM) with tamoxifen to induce PTEN deletion. Values are mean \pm S.E.M. ${ }^{\star \star} P<0.01$, by $t$-test analysis 
carcinogenesis, disabled TGF- $\beta$ signaling has been associated with loss of growth inhibition. ${ }^{35}$ In contrast, in endometrial cancer cell lines, TGF- $\beta$ promotes EMT and increases invasiveness. ${ }^{36}$

Mechanistically, we have demonstrated that the tumor suppressive functions of TGF- $\beta /$ Smad3 signaling are mediated by the regulation of PI3K/AKT signaling pathway (Figure 9). It has been reported that TGF- $\beta$ can directly activate PI3K/AKT signaling to regulate Smad3 nuclear translocation. $^{7}$ Here, we have demonstrated that TGF- $\beta$ induces Smad2/3 nuclear translocation, but independently of PI3K/AKT signaling. In the nucleus, Smad3, but not Smad2, binds to PTEN promoter and stimulates its transcription, which results in an inhibition of $\mathrm{PI} / \mathrm{K} / \mathrm{AKT}$ signaling. At the functional level, the inhibition of the PI3K/AKT pathway results in apoptotic cell death (Figure 9). The Smad3-dependent a

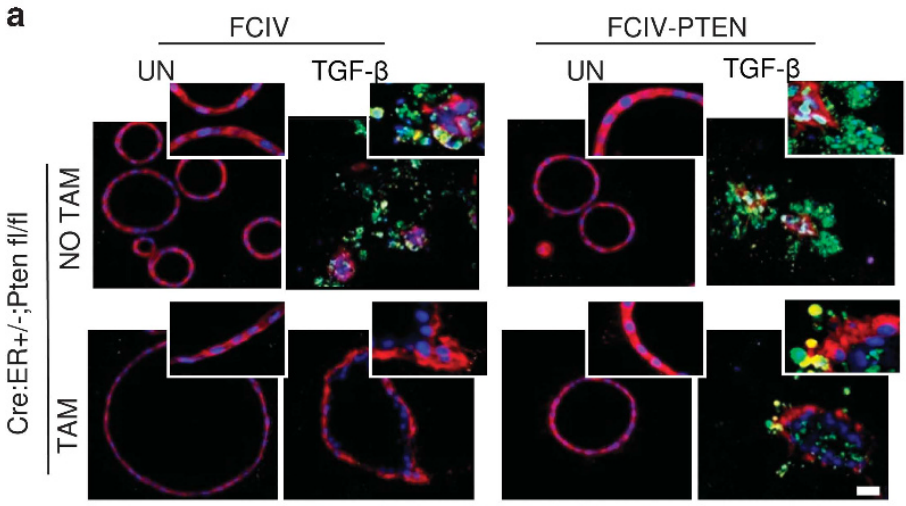

Cleaved Casp-3 / Tubulin / Hoechst

C

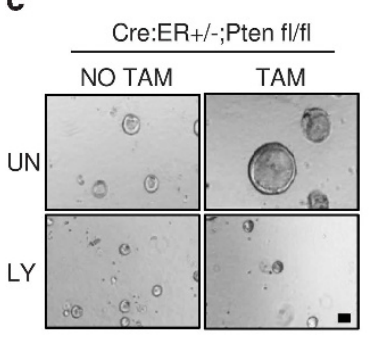

d UN

UN

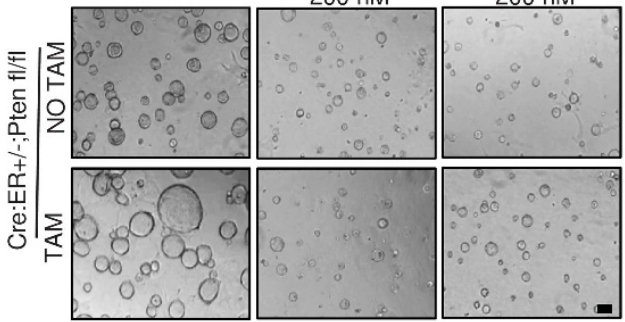

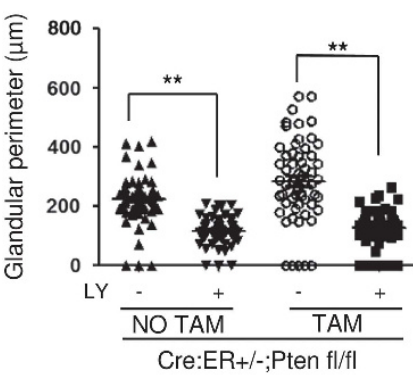

f

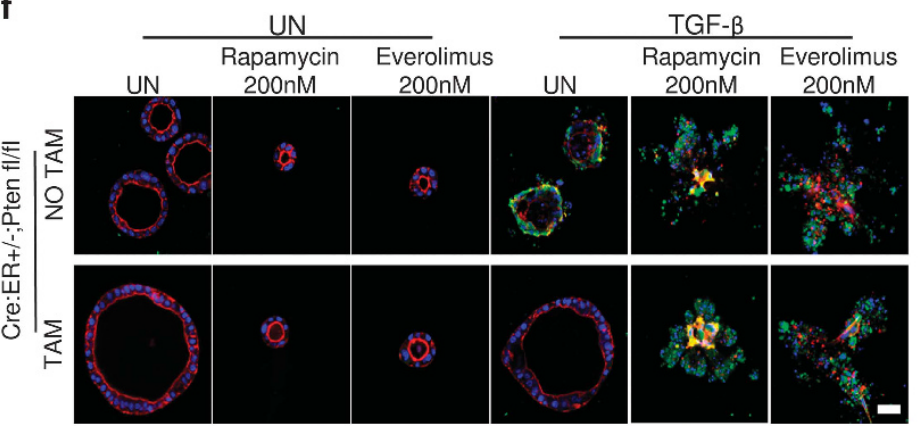

Cleaved Casp-3 / Tubulin / Hoechst b
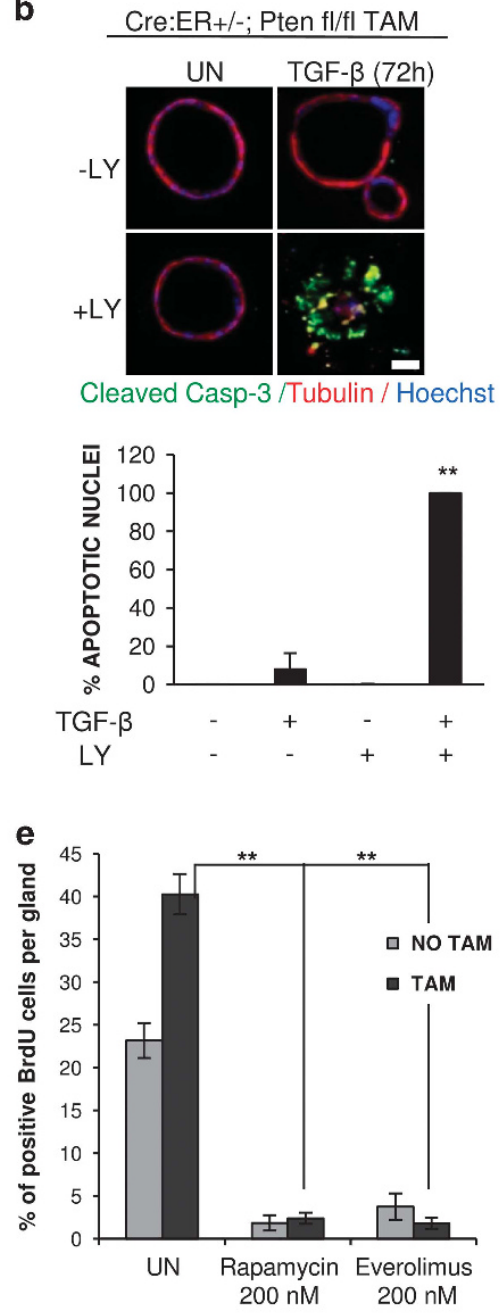

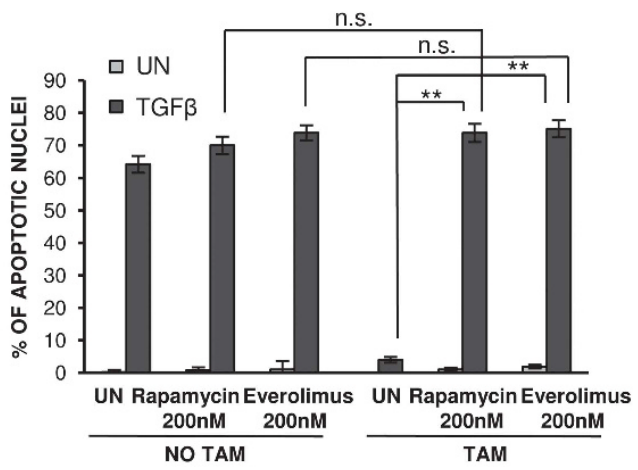


regulation of $\mathrm{PI} 3 \mathrm{~K} / \mathrm{AKT}$ pathway and its role in transducing tumor suppressive effects of TGF- $\beta$ signaling is supported by the results obtained in Smad3-deficient cells. Smad3 loss reduces PTEN transcription, resulting in enhanced PI3K/AKT signaling and increased cell proliferation and resistance to apoptosis (Figure 9). Hitherto, there are no existing evidences for a Smad3-dependent regulation of PTEN expression as a tumor suppressive mechanism for wild-type cells.

Although different mechanisms of cross talk between PI3K/ AKT and TGF- $\beta /$ Smad signaling have been reported, ${ }^{14-16}$ our findings uncover a novel mechanistic model to explain the antagonism between these two pathways. We have shown that PTEN loss leads to the activation of PI3K/AKT signaling, which enhances cell proliferation and impairs TGF- $\beta$-induced apoptosis and, therefore, TGF- $\beta$ suppressive functions. On the basis of our results, it is easy to speculate that loss of such tumor suppressive function may contribute to the development of endometrial hyperplasia and carcinoma in PTENdeficient mice.

The PI3K/AKT signaling pathway displays a high degree of complexity. PI3K can result in the activation of several downstream substrates apart from $\mathrm{AKT}^{37}$ Moreover, AKT phosphorylates many downstream substrates. ${ }^{38}$ Here, we have uncovered $\mathrm{PI} 3 \mathrm{~K} / \mathrm{AKT} / \mathrm{mTORC} 1$ as a signaling axis responsible for enhanced proliferation and resistance to TGF- $\beta$-induced apoptosis in PTEN knockout cells. It is worth mentioning that $\mathrm{mTORC} 1$ inhibitors are currently used in clinic as a therapeutic agent. ${ }^{39}$ Therefore, our findings, apart from new mechanistic insight, could have a therapeutic value.

Another issue that deserves discussion is the requirement of Smad2 or Smad4 to drive TGF- $\beta$-induced cellular responses. Most of cell responses triggered upon TGF- $\beta$ stimulation require association of $\mathrm{R}$-Smads (Smad2/3) with Smad4. However, increasing evidences demonstrate that Smad2 and Smad3 may have different roles in TGF- $\beta$ signaling. 40

To conclude, our study provides a new mechanistic and functional insight into the interplay between TGF- $\beta /$ Smad and PI3K/AKT signaling pathways to regulate important features of the tumoral phenotype such as cell proliferation and apoptosis.

\section{Materials and Methods}

Reagents and antibodies. Matrigel ( $\mathrm{rBM}$ ) was purchased from $\mathrm{BD}$ Biosciences (San Jose, CA, USA). Epidermal growth factor (EGF) an LY294002 were from Sigma-Aldrich (St. Louis, MO, USA), insulin-transferrin-sodium selenite (ITS) supplement and recombinant TGF- $\beta$ were from Invitrogen (Carlsbad, CA, USA). BisBenzimide H 33342 trihydrochloride (Hoechst), (Z)-4-Hydroxytamoxifen (TAM) (\#H6278), rhodamine-conjugated phalloidin were obtained from SigmaAldrich. Everolimus and Rapamycin were from Selleckchem (Houston, TX, USA). Antibodies to cleaved caspase-3 (\#9961), PTEN (\#9188), p-AKT (ser473) (\#4060), cleaved caspase-9 (\#9588) and p-Smad2 (Ser465/467)/Smad3 (Ser423/425) (\#8828) and Smad2 (D43B4) XP Rabbit mAb (\#5339) were from Cell Signaling Technology (Beverly, MA, USA). Antibodies against TGF- $\beta$ (\#SC:146), T $\beta$ RI (\#SC:398), T $\beta$ RII (\#SC:220 and \#Sc:400), Cyclin D1 (\#sc:20044), Histone H1 (\#sc:8030), Pan-AKT (\#SC:1618) and Smad4 (\#Sc:7966) were from Santa Cruz Biotechnology (Santa Cruz, CA, USA). Antibody to $\alpha$-tubulin (\#T9026) was obtained from Sigma-Aldrich; anti-lactate dehydrogenase (LDH)(\#100-1173) was from Rockland Immunochemicals Inc. (Limerick, PA, USA); anti-total Smad2/3 (\#610051) was purchased from BD Biosciences; Anti-Smad3 antibody - ChIP Grade (\#28379) was purchased from Abcam (Cambridge, UK). The TGF- $\beta$ superfamily type I receptor inhibitor SB431542 was obtained from Sigma-Aldrich. The concentrations and dilutions of all antibodies used in western blot analysis, immunofluorescence and immunohistochemistry are shown in Supplementary Table 1.

Lentiviral plasmid encoding PTEN was constructed by subcloning PTEN CDNA from PKR5-PTEN plasmid (obtained from Rafa Pulido's laboratory, Bilbao, Spain). Lentiviral plasmid encoding CA-AKT was obtained as a gift from Elisabeth Krizman and Michael Robinson laboratory (Philadelphia, PA, USA). The lentivrival plasmid pHR-cmv-nls CRE encoding Cre recombinase was from Didier Trono Laboratory (Lausanne, Switzerland) and obtained from Addgene (Cambridge, MA, USA) (plasmid \#12265).

Genetically modified mouse models. Mice were housed in a barrier facility and pathogen-free procedures were used in all mouse rooms. Animals were kept in a 12-h light-dark cycle with ad libitum access to a standard 2014 Teklad Global 14\% Protein Rodent Maintenance Diet (www.harlan.com) and water. The animal rooms were environmentally controlled $\left(20 \pm 2{ }^{\circ} \mathrm{C}\right.$, relative humidity $50 \pm 5 \%$ ). The study complied with Law 5/1995 and Act 214/1997 of the Autonomous Community (Generalitat of Catalonia) and EU Directive EEC 63/2010, and was approved by the Ethics Committee on Animal Experiments of the University of Lleida and the Ethics Commission in Animal Experimentation of the Generalitat de Catalunya. Floxed homozygous pten (C;129S4-Ptentm1Hwu/J; referred here as $\left.\mathrm{pten}^{\mathrm{fl} / \mathrm{fl}}\right)$, Cre-ER ${ }^{\top}\left(\mathrm{B} 6 . \mathrm{Cg}-\mathrm{Tg}\left(\mathrm{CAG}-\mathrm{Cre} / \mathrm{Esr}{ }^{*} 5 \mathrm{Amc} / \mathrm{J}\right)\right.$ mice were obtained from the Jackson Laboratory (Bar Harbor, ME, USA). Cre-ER ${ }^{T+1}$ pten ${ }^{\mathrm{f} / \mathrm{fl}}$ mice were bred in a mixed background (C57BL6; 129S4) by crossing pten ${ }^{\text {t/fli }}$ and Cre-ER ${ }^{T+1}$ mice. To obtain mice carrying both PTEN-floxed alleles $\left(\right.$ pten $\left.^{\mathrm{fl} / \mathrm{fl}}\right)$ and a single Cre-ER ${ }^{\mathrm{T}}\left(\mathrm{Cre}-\mathrm{ER}^{\mathrm{T}+1-}\right)$, Cre-ER ${ }^{\mathrm{T}+1}$ pten ${ }^{\mathrm{fl} /+}$ mice were backcrossed with pten $^{\mathrm{fl} / f \mid l}$ mice to obtain Cre-ER ${ }^{\mathrm{T}+1}$ pten $^{\mathrm{fl} / \mathrm{fl}}$ (referred here as Cre:ER+/ - ; Pten fl/fl). $\mathrm{Smad}^{\mathrm{f} / / \mathrm{fl}}$ and $\mathrm{Smad} 3^{\mathrm{ex} 8 / \mathrm{ex} 8}$ (referred here as Smad3 - / - ) mice with a C57BL/6

\footnotetext{
Figure 8 PI3K/AKT/mTORC1 inhibition reduces proliferation and restores TGF- $\beta$-induced apoptosis in PTEN-deficient cells. (a) Representative images of cleaved caspase-3 on Cre:ER+/ - ; Pten fl/fl 3D cultures treated (TAM) or not (NO TAM) with tamoxifen to induce PTEN deletion, infected with lentiviruses carrying PTEN cDNA (FCIV-PTEN) or the empty vector (FCIV) and treated or not (UN) with TGF- $\beta$ with $10 \mathrm{ng} / \mathrm{ml}$ for $36 \mathrm{~h}$. Magnification images of framed regions of the samples are shown to demonstrate apoptotic cell death. 3D cultures were also stained with tubulin and Hoechst to show glandular structure morphology and apoptotic nuclei. Scale bar: $25 \mu \mathrm{m}$. (b) Upper panel, representative images of cleaved caspase-3, tubulin and Hoechst staining on Cre:ER+/ - ; Pten fl/fl 3D cultures incubated with tamoxifen and treated with TGF- $\beta$ with $10 \mathrm{ng} / \mathrm{ml}$ for $72 \mathrm{~h}$ in the presence $(+L Y)$ or absence $(-L Y)$ of $L Y 294002$. Bottom, quantification of nuclei displaying positive immunostaining for cleaved caspase-3. Scale bar: $25 \mu \mathrm{m}$. ${ }^{* \star} P \leqslant 0.01$ (one-way ANOVA Tukey's multiple comparison test). (c) Representative phase contrast images and measurement of glandular perimeter of 3D cultures from Cre:ER+/ -; Pten fl/fl 3D cultures treated (TAM) or not (NO TAM) with tamoxifen to induce PTEN deletion in combination with (+LY) or without $(-L Y)$ LY294002. Errors bars represent mean \pm S.E.M. ${ }^{* *} P<0.01$ by $t$-test analysis. Scale bars: $50 \mu \mathrm{m}$. (d) Upper panel, representative phase contrast images and of 3D cultures from Cre:ER+/ - ; Pten fl/fl 3D cultures treated (TAM) or not (NO TAM) with tamoxifen and treated with $200 \mathrm{nM}$ of Rapamycin or Everolimus or left untreated (UN). Bottom, measurement of glandular perimeter of 3D cultures from Cre: $\mathrm{ER}+1-$; Pten fl/fl 3D cultures treated (TAM) or not (NO TAM) with tamoxifen and then with $200 \mathrm{nM}$ of Rapamycin or Everolimus or left UN. Errors bars represent mean \pm S.E.M.; ${ }^{* *} P<0.01$ by one-way ANOVA followed by the Tukey's multiple comparison test. Scale bar: $100 \mu \mathrm{m}$. (e) Quantification of BrdU-positive cells 3D cultures from Cre:ER+/ $-;$ Pten fl/ fl 3D cultures treated (TAM) or not (NO TAM) with tamoxifen and then with $200 \mathrm{nM}$ of Rapamycin or Everolimus or UN. Values are mean and error bars represent mean \pm S.E.M. ${ }^{* *} P<0.01$ by one-way ANOVA, followed by the Tukey's multiple comparison test. (f) Representative images and quantification of caspase-3-positive cells 3D cultures from Cre: $\mathrm{ER}+\mathrm{l}-$; Pten fl/fl 3D cultures treated (TAM) or not (NO TAM) with tamoxifen and then with $200 \mathrm{nM}$ of Rapamycin or Everolimus alone or in combination with $10 \mathrm{ng} / \mathrm{ml}$ of TGF- $\beta$ for $36 \mathrm{~h}$. Cultures were immunostained with tubulin to show glandular structures. Nuclei were counterstained with Hoechst to show apoptotic nuclear morphology. Values are mean \pm S.E.M. ${ }^{* *} P<0.01$, by two-way ANOVA, followed by the Bonferroni test. NS, not significant
} 


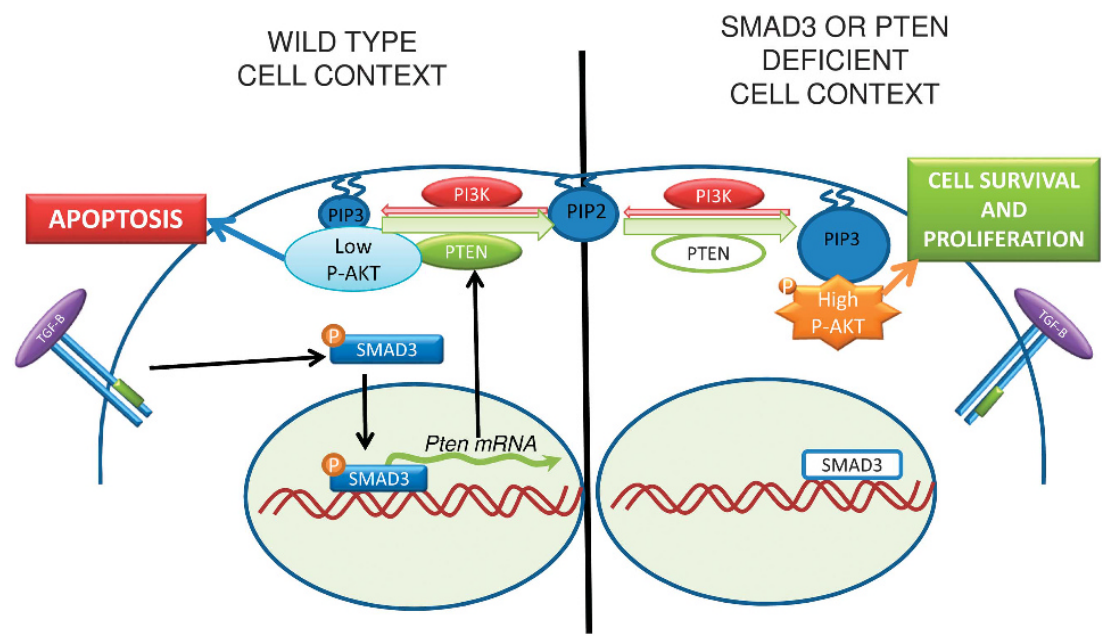

Figure 9 A mechanistic model for PTEN-Smad3 regulatory loop. In a wild-type cell context, TGF- $\beta$ acts as a tumor suppressor by inducing apoptosis. TGF- $\beta$ stimulation leads to nuclear translocation of Smad2/3. In the nucleus, Smad3 is required to transcribe PTEN, which inhibits PI3K/AKT signaling and enables TGF- $\beta$-induced apoptosis. In Smad3- or PTEN-deficient cells context TGF- $\beta$-induced upregulation of PTEN mRNA is impaired, resulting in enhanced PI3K/AKT activation, which leads to increased proliferation and hampers apoptosis triggered by TGF- $\beta$

background were provided by Dr. Akihiko Yoshimura (Keio University School of Medicine). ${ }^{41}$ Mice were genotyped by earmarking and DNA was isolated from tail tissue in proteinase $\mathrm{K}$ lysis buffer. PCR was carried out with GoTaq Polymerase (Promega, Madison, WI, USA) using different pairs of primers for each gene. pten ${ }^{f / f t}$ forward primer 5'-CAAGCACTCTGCGAACTGAG-3' and reverse primer 5'-AAGT TTTTGAAGGCAAGATGC-3'; Cre-ER ${ }^{\mathrm{T}+1}$ - forward primer $5^{\prime}$-ACGAACCTGGTCGA AATCGTGCG-3' and reverse primer 5'-CGGTCGATGCAACGAGTGATGAG-3'; Smad3 - / - using a common forward primer 5 '-CCACTTCATTGCCATATGCCCTG $-3^{\prime}$ and the Smad3+/+ reverse primer $5^{\prime}-$ CCCGAACAGTTGGATTCACACA-3' or the Smad3 - / - reverse primer 5'-CCAGACTGCCTTGGGAAAAGC-3'. No statistical method was used to predetermine sample size. No samples and animals were excluded from the analysis. The investigators were not blinded to allocation during experiments or outcome assessment.

Isolation of endometrial epithelial cells. The isolation of endometrial epithelial cells was performed using a method previously described with minor modifications. ${ }^{42}$ In brief, uterine horns were dissected from mice of 3-4 weeks old. Uteri were washed with Hank's balanced salt solution (HBSS) and digested with trypsin (Invitrogen). After trypsin digestion, epithelial sheets were squeezed out of the uterine pieces. Epithelial sheets were washed twice with phosphate-buffered saline (PBS) and resuspended in $1 \mathrm{ml}$ of DMEM/F12 medium (Invitrogen) supplemented with $1 \mathrm{mM}$ HEPES (Sigma-Aldrich), 1\% of penicillin/streptomycin (Sigma-Aldrich) and fungizone (Invitrogen) (basal medium). Epithelial sheets were mechanically disrupted in basal medium. Cells were diluted in basal medium containing $2 \%$ dextran-coated charcoal-stripped (DCC-S) serum (Hyclone, Logan, UT, USA) and plated in culture dishes (BD Falcon, Bedford, MA, USA). Cells were cultured for $24 \mathrm{~h}$ in an incubator at $37^{\circ} \mathrm{C}$ with saturating humidity and $5 \% \mathrm{CO}_{2}$.

Three-dimensional glandular cultures. Culture of endometrial epithelial cells in $3 \mathrm{D}$ cultures was performed as described previously ${ }^{42}$ with minor modifications. Freshly isolated endometrial epithelial cells were plated in 24- or 6-well plastic culture plates (BD Biosciences). When required, PTEN deletion in endometrial cells isolated from Cre:ER+l-; Pten fl/fl mice was induced by the addition of $0.5 \mu \mathrm{g} / \mathrm{ml}$ of tamoxifen in culture medium. To generate Smad2 - / endometrial cells isolated from Smad2 $2^{\mathrm{fl} / \mathrm{ll}}$ mice, cells were infected with lentiviral plasmid encoding the Cre recombinase pHR-cmv-nls CRE.

After $24 \mathrm{~h}$, wild-type or genetically modified cells were washed with HBSS and incubated with trypsin/EDTA solution (Sigma-Aldrich) for $5 \mathrm{~min}$ at $37^{\circ} \mathrm{C}$. Trypsin activity was stopped by adding Dulbecco's modified Eagle's medium (DMEM) (Invitrogen) containing 10\% fetal bovine serum (FBS) (Invitrogen). Clumps of 2-8 cells were obtained. Cells were centrifuged at $200 \times g$ for 3 min and diluted in basal medium containing $3 \%$ Matrigel to obtain $4 \times 10^{4}$ cell clumps per ml. For immunofluorescence, cells were seeded in a volume of $40 \mu \mathrm{l}$ per well in 96-well plates with micro-clear bottom (Gr Greiner Bio-One, Longwood, FL, USA). For western blotting and real-time qPCR, cells were placed in a volume of $200 \mu \mathrm{l}$ in 24well plates (BD Biosciences). In all cases, $24 \mathrm{~h}$ after plating, medium was replaced by basal medium supplemented with $5 \mathrm{ng} / \mathrm{ml}$ EGF, $1: 100$ dilution of ITS supplement and $3 \%$ of fresh Matrigel. Medium was replaced every 2-3 days.

When 3D culture was established from genetically modified mice models, 3-10 pooled uteri were used to obtain endometrial epithelial cells. No statistical method was used to predetermine sample size. No specific methods for randomization were applied.

Viral production, infection and in vitro transfection conditions. Oligonucleotides to produce plasmid-based shRNA were cloned into the FSV vector using Agel-BamHI restriction sites. shRNA target sequences were: non-targeting sequence (control shRNA sequence inserted in FSV plasmid and used as control throughout) 5'-CCTAAGGTTAAGTCGCCCTC-3'; Smad2.1: 5'-GGTGTTCAATCG CATACTAT-3'; Smad2.2: 5'-CGGTTAGATGAGCTTGAGAAA-3'; Smad3.1: 5'-CTG TCCAATGTCAACCGGAAT-3'; Smad3.2: 5'-CATCCGTATGAGCTTCGTCAA-3'.

Production of small hairpin RNA against Smad3 was achieved by transfecting HEK-293T packaging cells with linear PEI $(40 \mu \mathrm{M})$ in combination with lentiviral plasmids (shRNA Smad3.1 and Smad3.2) and (psPAX2 packaging and pMD2G envelope) helper plasmids at 1:1:1 ratio, respectively. Similarly, the same conditions were used to produce FCIV-PTEN or CA-AKT carrying lentiviruses.

Four hours after transfection, packaging cells were cultured with DMEM supplemented with $10 \%$ FBS, $1 \mathrm{mmol} / \mathrm{HEPES}$ (Sigma-Aldrich), $1 \mathrm{mmol} / \mathrm{l}$ sodium pyruvate (Sigma-Aldrich), $2 \mathrm{mmo} / / \mathrm{L}$-glutamine (Sigma-Aldrich) and $1 \%$ of penicillin/ streptomycin (Sigma-Aldrich) for 3-4 days; afterwards the medium containing the viral particles was collected, centrifuged for $5 \mathrm{~min}$ at $200 \times \mathrm{g}$ and filtered through a $0.45 \mu \mathrm{M}$ filter (Millipore, Temecula, CA, USA) and concentrated using Vivaspin concentrators (Sartorius Stedim Biotech $\mathrm{GmbH}$, Gottingen, Germany). The concentrated medium containing lentiviral particles was added to growing primary cultures in basal medium. Cells were incubated for $24 \mathrm{~h}$. After this period, the medium was replaced with fresh medium and cells were grown regularly to allow endogenous protein knockdown or protein overexpression.

Total RNA extraction, reverse transcriptase-PCR and quantitative real-time. For RT-qPCR, total RNA was extracted from the uterine endometrium or 3D cultures using the RNeasy Total RNA kit (Qiagen, Valencia, CA, USA) and CDNA was generated using the High-Capacity cDNA Archive Kit (Applied Biosystems, Foster City, CA, USA). The CDNA product was used as a template for subsequent PCR amplifications for apoptotic and anti-apoptotic members of the Bcl2 family. Primers were designed to be complementary to the published mouse sequences and include as many known variants as possible. Glyceraldehyde-3phosphate dehydrogenase (GAPDH) and $\beta$-actin were used as a control 
housekeeping genes. Primer sequences, product sizes and PCR conditions were as follows: (5'-3') GAPDH: Forward (Fw): TGGCCTTCCGTGTTCCTAC, Reverse (Rv): GAGTTGCTGTTGAAGTCGCA, length (L): $178 \mathrm{bp}$ and temperature (T): $63^{\circ} \mathrm{C} ; \beta$ actin: Fw GGCTGTATTCCCCTCCATCG, Rv; CCAGTTGGTAACAATGCCATGT, (L): 154 bp (T): $64^{\circ} \mathrm{C}$; BIM: Fw GACAGAACCGCAAGGTAATCC, Rv; ACTTGTCAC AACTCATGGGTG, (L): $215 \mathrm{bp}$ (T): $62^{\circ} \mathrm{C} ; \quad$ BMF: Fw CAGAGACTCTTTT ACGGCAACG, Rv; ACTGGTCTGCAATACACTGAAG, (L): $157 \mathrm{bp}$ (T): $62^{\circ} \mathrm{C}$; NOXA: Fw CTCAGGAAGATCGGAGACAAAGT, Rv; GAGTTGAGCACACTCGT CCTT, (L): 156 bp (T): $63^{\circ} \mathrm{C}$; BAK: Fw CAACCCCGAGATGGACAACTT, Rv; CGTAGCGCCGGTTAATATCAT, (L): $101 \mathrm{bp}$ (T): $62^{\circ} \mathrm{C}$; BAK: Fw CAACCCC GAGATGGACAACTT, Rv; CGTAGCGCCGGTTAATATCAT, (L): 101 bp (T): $62^{\circ} \mathrm{C}$; BAX: Fw TGAAGACAGGGGCCTTTTTG, Rv; AATTCGCCGGAGACACTCG, (L): 140 bp (T): $62{ }^{\circ} \mathrm{C}$; PUMA: Fw TGTATCCTGCAGCCTTTGC, Rv; ACGGGCGACTCT AAGTGCT, (L): 222 bp (T): $61^{\circ} \mathrm{C}$; BIK: Fw GAGCCTGTGAGAGACGTGG, Rv; CGAGTCTGTGTATAGCAATCCCA, (L): $148 \mathrm{bp}(\mathrm{T}): 63^{\circ} \mathrm{C}$ and BAD: Fw AGGGA TGGAGGAGGAGCTTA, Rv; CCCACCAGGACTGGATAATG, (L): 212 bp (T): $62^{\circ} \mathrm{C}$.

For RT-qPCR using SYBR green, $5 \mu$ of cDNA was combined with Maxima SYBR Green qPCR Master Mix (2x) (Thermo Scientific, Rockford, IL, USA), and 10 pmol of each primer in a final volume of $20 \mu \mathrm{l}$. After initial denaturation at $95^{\circ} \mathrm{C}$ for $15 \mathrm{~min}$, the reaction mixture was subjected to 35 successive cycles of denaturation at $95^{\circ} \mathrm{C}$ for $15 \mathrm{~s}$, annealing at (indicated temperatures) for $15 \mathrm{~s}$, extension at $72{ }^{\circ} \mathrm{C}$ for $30 \mathrm{~s}$ and reading at $81^{\circ} \mathrm{C}$. Product purity was confirmed by dissociation curves, and random samples were subjected to agarose gel electrophoresis. Controls containing no template DNA were included in all assays, yielding no consistent amplification. The relative expression levels of each target were calculated based on the cycle threshold method.

For RT-qPCR assays using Taqman probes, the CDNA was amplified by heating to $95{ }^{\circ} \mathrm{C}$ for $10 \mathrm{~min}$, followed by $40 \mathrm{PCR}$ cycles of $95^{\circ} \mathrm{C}$ for $15 \mathrm{~s}$ and $60^{\circ} \mathrm{C}$ for $1 \mathrm{~min}$ using the ABI Prism 7900 Sequence Detection System (Applied Biosystems) and Promega GoTaq aPCR Master Mix. Relative mRNA expression levels were calculated using the $2 \Delta \Delta \mathrm{Ct}$ method and are presented as ratios to the housekeeping gene GAPDH. Taqman technology from Applied Biosystems was used for real-time RT-qPCR analyses. Probes: GAPDH, Mm99999915_g1; PTEN, Mm00477208_m1; Smad2, Mm00487530_m1; Smad3, Mm01170760_m1; BIM, Mm00437796_m1. The number of cycles needed to reach the crossing point for each sample was used to calculate the amount of each product using the 2-CP method. Each sample pool was amplified in triplicate using GAPDH for normalization.

Chromatin immunoprecipitation. Endometrial epithelial cells in 3D culture (untreated and cells treated with TGF $\beta 10 \mathrm{ng} / \mathrm{ml}$ for $30 \mathrm{~min}$ ) were crosslinked for $15 \mathrm{~min}$ at room temperature by adding $1 / 10$ volumes of fresh $11 \%$ formaldehyde solution. Subsequently we quenched formaldehyde by adding glycine for $15 \mathrm{~min}$. Cells were washed twice in ice-cold PBS and lysed. Lysate was divided in volume of $100 \mu \mathrm{l}$ and sonicated. Sonication conditions were: 4 cycles of 30" sonication and 20" pause at $20 \%$ amplitude. This protocol was repeated for three consecutive times on ice. Finally the solution was pelleted and the supernatant transferred into a new collecting tube and saved at $-80^{\circ} \mathrm{C}$. At this point we saved $10 \mu \mathrm{l}$ as a whole-cell extract input. In parallel we conjugated the anti-Smad2/3 antibody to the beads. Anti-Smad2/3 conjugated beads were collected and washed with blocking buffer. About $100 \mu \mathrm{l}$ of nuclear extracts were added to the bead solution and incubated overnight at $4{ }^{\circ} \mathrm{C}$ on a rotator. After $12 \mathrm{~h}$, beads were collected, incubated and washed with low and high salt buffer, next with $\mathrm{LiCl}$ wash buffer and finally with TE buffer. Beads were centrifuged and resuspended with elution buffer. The solution was incubated at $65^{\circ} \mathrm{C}$ in a heat block for 15 min. Afterwards, elution buffer was added to input. Input and pellet were incubated in the oven overnight at $65^{\circ} \mathrm{C}$. Lysates were centrifuged and the supernatants transferred into new tubes. Supernatants were digested and incubated at $37^{\circ} \mathrm{C}$ for $2 \mathrm{~h}$ in TE buffer containing RNAseA and subsequently digested in proteinase $\mathrm{K}$ and incubated for $2 \mathrm{~h}$ at $55^{\circ} \mathrm{C}$. To proceed with DNA extraction, we added phenol/chloroform/isoamyl alcohol into a phaselock tube. Reverse crosslinked samples were transferred to the phaselock tubes, and centrifuged at top speed for $10 \mathrm{~min}$ at RT. Aqueous phase was transferred to a new tube and the DNA was precipitated by adding $\mathrm{NaCl}$, glycogen and $100 \% \mathrm{EtOH}$. We incubated the samples at $-20{ }^{\circ} \mathrm{C}$ for $4 \mathrm{~h}$ and then centrifuged the samples at top speed. The pellets were washed with $80 \% \mathrm{EtOH}$. Samples were spinned again and the pellet was resuspended in TE buffer and incubated at $65^{\circ} \mathrm{C}$ on a heating block for $15 \mathrm{~min}$.

Immunoprecipitated DNA was subjected to GPCR analysis as described in the previous section. Primers used for GPCR were: PTEN PROMOTER $-1 /-500$ $\left(5^{\prime}-3^{\prime}\right)$ (F TCGGAAAGCCGGAGGGGAG, R GTGTCTCCCGCGTGGGTCA); PTEN PROMOTER - 501/-986 (F TGACCCACGCGGGAGACAC, R GGCCTGGGAG
GGCTCAAAG); PTEN PROMOTER - 987/-1459 (F CTTTGAGCCCTCC CAGGCC, R CAACCGTGGGAGAAGAGGC).

Western blotting. Cells were washed with cold PBS and lysed with lysis buffer ( $2 \% \mathrm{SDS}, 125 \mathrm{mmol} / \mathrm{Tris}-\mathrm{HCl}, \mathrm{pH}$ 6.8). Protein concentrations were determined by loading on a $10 \%$ acrylamide gel, transferring to polyvinylidene difluoride membranes (Millipore), and blotting with anti-tubulin antibody. Band density was determined by using Image Lab 4.0.1 software (Bio-Rad laboratories, Richmond, CA, USA). Equal amounts of proteins were subjected to SDS-polyacrylamide gel electrophoresis and transferred to polyvinylidene difluoride membranes. Nonspecific binding was blocked by incubation with TBST $(20 \mathrm{mM}$ Tris- $\mathrm{HCl}(\mathrm{pH} 7.4)$, $150 \mathrm{mM} \mathrm{NaCl}$ and $0.1 \%$ Tween 20) plus $5 \%$ of non-fat milk. Membranes were incubated with the primary antibodies overnight at $4^{\circ} \mathrm{C}$ and for $1 \mathrm{~h}$ room temperature with secondary horseradish peroxidase (1:10 000 in TBST). Signal was detected with ECL Advance (Amersham-Pharmacia, Little Chalfont, Buskinghamshire, UK) and SuperSignal West Femto Trial Kit (Thermo Scientific).

Bromodeoxyuridine incorporation assay. For the determination of DNA and after the indicated treatments, 3D cultures were incubated with $3 \mathrm{ng} / \mathrm{ml}$ of 5-bromodeoxyuridine (BrdU, Sigma-Aldrich) during $15 \mathrm{~h}$ and then fixed with $4 \%$ paraformaldehyde for $20 \mathrm{~min}$. DNA denaturation was performed with $2 \mathrm{~mol} / \mathrm{HCl}$ for 30 min. Afterwards, neutralization was done with $0.1 \mathrm{~mol} / / \mathrm{Na}_{2} \mathrm{~B}_{4} \mathrm{O}_{7}(\mathrm{pH} 8.5)$ for 2 min and after rinsed three times with PBS. Subsequently, block cells in PBS solution containing $5 \%$ horse serum, $5 \%$ FBS, $0.2 \%$ glycine and $0.1 \%$ Triton X-100 for $1 \mathrm{~h}$. Next, cells were incubated in anti-BrdU primary monoclonal antibody (DAKO, Glostrup, Denmark), and fluorescein isothiocyanate-conjugated anti-mouse secondary antibody. Nuclei were counterstained with $5 \mathrm{mg} / \mathrm{ml} \mathrm{Hoechst} 33258$ and cells were visualized under an epifluorescence microscope (Leica Microsystems, Wetzlar, Germany). Nuclei were counterstained with $5 \mu \mathrm{g} / \mathrm{ml}$ Hoechst 33258. Cells were visualized under a confocal microscope. BrdU-positive nuclei were scored and divided by the total number of cells (visualized by Hoechst staining). The results are expressed as a percentage of BrdU-positive cells.

Immunohistochemical study. After killing, mice uteri were excised, flushed with PBS, fixed in $10 \%$ neutral-buffered formalin and embedded in paraffin. Sections $(4-5 \mu \mathrm{m})$ were stained with hematoxylin and eosin for histopathology. Mice uterus and TMA blocks from human tissue samples were sectioned at a thickness of $3 \mu \mathrm{m}$, dried for $1 \mathrm{~h}$ at $65^{\circ} \mathrm{C}$ before pre-treatment procedure of deparaffinization, rehydration and epitope retrieval in the Pre-Treatment Module, PT-LINK (DAKO) at $95{ }^{\circ} \mathrm{C}$ for $20 \mathrm{~min}$ in $50 \times$ Tris/EDTA buffer, pH 9. Before staining the sections, endogenous peroxidase was blocked. The antibodies used were against $6 \mathrm{H} 2.1$ and p-AKT. After incubation, the reaction was visualized with the EnVisionTM FLEX Detection Kit (DAKO) for PTEN and EnVisionTM FLEX+ rabbit (LINKER) Detection Kit (DAKO) for p-AKT using diaminobenzidine chromogen as a substrate. Sections were counterstained with hematoxylin. Appropriate negative controls including no primary antibody were also tested.

Immunohistochemical results were evaluated by following uniform pre-established criteria. Immunostaining was graded semi-quantitatively by considering the percentage and intensity of the staining. A histological score was obtained from each sample and values ranged from 0 (no immunoreaction) to 300 (maximum immunoreactivity). The score was obtained by applying the following formula, Histoscore $=1 \times(\%$ light staining) $+2 \times(\%$ moderate staining $)+3 \times$ ( $\%$ strong staining). The histological score was also used for evaluation of cytosolic and nuclear staining intensity.

To support the scoring of immunohistochemistry an automated imaging system, the ACIS III Instrument (DAKO), was also used. An intensity score, which ranged from 60 to 255 , was obtained from four different areas of each sample.

Immunofluorescence. Endometrial epithelial cells in 3D cultures were fixed with formalin for $5 \mathrm{~min}$ at room temperature, and washed twice with PBS. Depending on primary antibody, cells were permeabilized with $0.2 \%$ Triton $(T) X-100$ in PBS for 10 min or with $100 \%$ methanol (Me) for 2 min. Next, cultures were incubated overnight at $4{ }^{\circ} \mathrm{C}$ with the indicated dilutions of antibodies: cleaved caspase-3 $(\mathrm{T}), \alpha$-tubulin $(\mathrm{T})$, total Smad2/3 (T), T $\beta R I(T), T \beta R I I$ (T) and anti-Smad4 (Me). After 1 day, cells were washed twice with PBS and incubated with PBS containing $5 \mu \mathrm{g} / \mathrm{ml}$ of Hoechst 33342 and a 1:500 dilution of Alexa Fluor secondary anti-mouse or anti-rabbit antibodies for $4 \mathrm{~h}$ at room temperature. For double-immunofluorescence staining, cells were incubated with the second round of primary and secondary antibodies. In all doubleimmunofluorescence stains, first and second primary antibodies were from a different isotype. Immunofluorescence staining was visualized and analyzed using confocal 
microscopy (model FV1000, Olympus, Tokyo, Japan) with the $\times 10$ and the oilimmersion $\times 60$ magnification objectives. Analysis of images was obtained with Fluoview FV100 software (Olympus).

Confocal imaging and evaluation of Spheroid perimeter. Images of endometrial epithelial spheroids were captured and digitized with a confocal microscope (Fluoview FV1000, Olympus). Epithelial perimeter analysis was processed by image analysis software (ImageJ version 1.46r; $\mathrm{NIH}$, Bethesda, $M D, U S A)$, generating binary images of the spheroids as previously described. ${ }^{43,44}$

Statistical analysis. The normality of the distribution of experiments was assessed by Kolmogorov-Smirnov test. No statistical method was used to predetermine sample size. Statistical analysis was performed with GraphPad Prism 6.0 (La Jolla, CA, USA). Differences between two groups were assessed by Student's t-test (unpaired or paired as needed depending on the study design). Differences between more than two groups were assessed by one-way ANOVA, followed by the Tukey's multiple comparison test or two-way ANOVA, followed by the Bonferroni post hoc comparison test. A $P<0.05$ was considered statistically significant. All data examined are expressed as mean \pm S.E.M.

\section{Conflict of Interest}

The authors declare no conflict of interest.

Acknowledgements. The study was supported by grants SAF2016-80157-R from Ministerio de Economía y Competitividad, Pl13/00263 and Pl13/01701 from Fondo de Investigaciones Sanitarias del Instituto de Salud Carlos III cofinanciado por Fondo Europeo de Desarrollo Regional (FEDER) ('Una manera de hacer Europa'), Red Temática de investigación en Cáncer RD12/0036/0013 and Red de Oncología (CIBERONC). Grups consolidats de la Generalitat de Catalunya (2009SGR794), Fundació La Marató de TV3, Grupos estables AECC, Catalunya contra el cáncer and programa de intensificación de la investigación, Instituto Carlos III. We would like to thank Mónica Domingo, Montse Ortrega and Marta Hereu for their technical support.

1. Massagué J. TGF $\beta$ signalling in context. Nat Rev Mol Cell Biol 2012; 13: 616-630.

2. Massagué J, Seoane J, Wotton D. Smad transcription factors. Genes Dev 2005; 19 : 2783-2810.

3. Heldin C-H, Moustakas A. Role of Smads in TGF $\beta$ signaling. Cell Tissue Res 2012; 347: 21-36.

4. Schmierer B, Hill CS. TGFbeta-SMAD signal transduction: molecular specificity and functional flexibility. Nat Rev Mol Cell Biol 2007; 8: 970-982.

5. Itoh S, ten Dijke P. Negative regulation of TGF- $\beta$ receptor/Smad signal transduction. Curr Opin Cell Biol 2007; 19: 176-184.

6. Mu Y, Gudey SK, Landström M. Non-Smad signaling pathways. Cell Tissue Res 2012; 347: 11-20.

7. Zhang YE. Non-Smad pathways in TGF-beta signaling. Cell Res 2009; 19: 128-139.

8. Padua D, Massagué J. Roles of TGFbeta in metastasis. Cell Res 2009; 19: 89-102.

9. Massagué J. TGFbeta in cancer. Cell 2008; 134: 215-230.

10. Xu J, Lamouille S, Derynck R. TGF-beta-induced epithelial to mesenchymal transition. Cell Res 2009; 19: 156-172.

11. Heldin C-H, Landström M, Moustakas A. Mechanism of TGF-beta signaling to growth arrest, apoptosis, and epithelial-mesenchymal transition. Curr Opin Cell Biol 2009; 21: 166-176.

12. Zhang $L$, Zhou F, ten Dijke P. Signaling interplay between transforming growth factor- $\beta$ receptor and PI3K/AKT pathways in cancer. Trends Biochem Sci 2013; 38: 612-620.

13. Song MS, Salmena L, Pandolfi PP. The functions and regulation of the PTEN tumour suppressor. Nat Rev Mol Cell Biol 2012; 13: 283-296.

14. Conery AR, Cao Y, Thompson EA, Townsend CM, Ko TC, Luo K. Akt interacts directly with Smad3 to regulate the sensitivity to TGF-beta induced apoptosis. Nat Cell Biol 2004; 6: 366-372.

15. Remy I, Montmarquette A, Michnick SW. PKB/Akt modulates TGF-beta signalling through a direct interaction with Smad3. Nat Cell Biol 2004; 6: 358-365.

16. Seoane J, Le H-V, Shen L, Anderson SA, Massagué J. Integration of Smad and Forkhead pathways in the control of neuroepithelial and glioblastoma cell proliferation. Cell 2004; 117 211-223.

17. Ding Z, Wu C-J, Chu GC, Xiao Y, Ho D, Zhang J et al. SMAD4-dependent barrier constrains prostate cancer growth and metastatic progression. Nature 2011; 470: 269-273.
18. Qin J, Wu S-P, Creighton CJ, Dai F, Xie X, Cheng C-M et al. COUP-TFIl inhibits TGF- $\beta$-induced growth barrier to promote prostate tumorigenesis. Nature 2013; 493: 236-240.

19. Yu M, Trobridge $P$, Wang $Y$, Kanngurn S, Morris SM, Knoblaugh S et al. Inactivation of TGF- $\beta$ signaling and loss of PTEN cooperate to induce colon cancer in vivo. Oncogene 2014; 33: 1538-1547.

20. Li Q. Transforming growth factor $\beta$ signaling in uterine development and function. J Anim Sci Biotechnol 2014; 5: 52.

21. Li Q, Agno JE, Edson MA, Nagaraja AK, Nagashima T, Matzuk MM. Transforming growth factor $\beta$ receptor type 1 is essential for female reproductive tract integrity and function. PLOS Genet 2011; 7: e1002320.

22. Gao Y, Li S, Li Q. Uterine epithelial cell proliferation and endometrial hyperplasia: evidence from a mouse model. Mol Hum Reprod 2014; 20: 776-786.

23. Yeramian A, Moreno-Bueno G, Dolcet X, Catasus L, Abal M, Colas E et al. Endometrial carcinoma: molecular alterations involved in tumor development and progression. Oncogene 2013; 32: 403-413.

24. Hollander MC, Blumenthal GM, Dennis PA. PTEN loss in the continuum of common cancers, rare syndromes and mouse models. Nat Rev Cancer 2011; 11: 289-301.

25. Eritja N, Dolcet X, Matias-Guiu X. Three-dimensional epithelial cultures: a tool to model cancer development and progression. Histol Histopathol 2013; 28: 1245-1256.

26. Pardali K, Moustakas A. Actions of TGF-beta as tumor suppressor and pro-metastatic factor in human cancer. Biochim Biophys Acta 2007; 1775: 21-62.

27. Siegel PM, Massagué J. Cytostatic and apoptotic actions of TGF-beta in homeostasis and cancer. Nat Rev Cancer 2003; 3: 807-821.

28. Ramesh S, Wildey GM, Howe PH. Transforming growth factor beta (TGFbeta)-induced apoptosis: the rise \& fall of Bim. Cell Cycle 2009; 8: 11-17.

29. Ramjaun AR, Tomlinson S, Eddaoudi A, Downward J. Upregulation of two BH3-only proteins, Bmf and Bim, during TGF beta-induced apoptosis. Oncogene 2007; 26: 970-981.

30. Wildey GM, Patil S, Howe PH. Smad3 potentiates transforming growth factor beta (TGFbeta )-induced apoptosis and expression of the BH3-only protein Bim in WEHI 231 B lymphocytes. J Biol Chem 2003; 278: 18069-18077.

31. Ramesh S, Qi X-J, Wildey GM, Robinson J, Molkentin J, Letterio J et al. TGF beta-mediated BIM expression and apoptosis are regulated through SMAD3-dependent expression of the MAPK phosphatase MKP2. EMBO Rep 2008; 9: 990-997.

32. Wiener Z, Band AM, Kallio P, Högström J, Hyvönen V, Kaijalainen S et al. Oncogenic mutations in intestinal adenomas regulate Bim-mediated apoptosis induced by TGF- $\beta$. Proc Natl Acad Sci USA 2014; 111: E2229-E2236.

33. Piestrzeniewicz-Ulanska D, Brys M, Semczuk A, Rechberger T, Jakowicki JA, Krajewska WM. TGF-beta signaling is disrupted in endometrioid-type endometrial carcinomas. Gynecol Oncol 2004; 95: 173-180.

34. Mhawech-Fauceglia P, Kesterson J, Wang D, Akers S, DuPont NC, Clark K et al. Expression and clinical significance of the transforming growth factor- $\beta$ signalling pathway in endometrial cancer. Histopathology 2011; 59: 63-72.

35. Parekh TV, Gama P, Wen X, Demopoulos R, Munger JS, Carcangiu M-L et al. Transforming growth factor beta signaling is disabled early in human endometrial carcinogenesis concomitant with loss of growth inhibition. Cancer Res 2002; 62: 2778-2790.

36. Muinelo-Romay L, Colas E, Barbazan J, Alonso-Alconada L, Alonso-Nocelo M, Bouso M et al. High-risk endometrial carcinoma profiling identifies TGF-1 as a key factor in the initiation of tumor invasion. Mol Cancer Ther 2011; 10: 1357-1366.

37. Bayascas JR. Dissecting the role of the 3-phosphoinositide-dependent protein kinase-1 (PDK1) signalling pathways. Cell Cycle 2008; 7: 2978-2982.

38. Manning BD, Cantley LC. AKT/PKB signaling: navigating downstream. Cell 2007; 129: 1261-1274.

39. Rodon J, Dienstmann R, Serra V, Tabernero J. Development of PI3K inhibitors: lessons learned from early clinical trials. Nat Rev Clin Oncol 2013; 10: 143-153.

40. Brown KA, Pietenpol JA, Moses HL. A tale of two proteins: differential roles and regulation of Smad2 and Smad3 in TGF-beta signaling. J Cell Biochem 2007; 101: 9-33.

41. Sugiyama Y, Kakoi K, Kimura A, Takada I, Kashiwagi I, Wakabayashi Y et al. Smad2 and Smad3 are redundantly essential for the suppression of iNOS synthesis in macrophages by regulating IRF3 and STAT1 pathways. Int Immunol 2012; 24: 253-265.

42. Eritja N, Llobet D, Domingo M, Santacana M, Yeramian A, Matias-Guiu X et al. A novel three-dimensional culture system of polarized epithelial cells to study endometrial carcinogenesis. Am J Pathol 2010; 176: 2722-2731.

43. Eritja N, Mirantes C, Llobet D, Masip G, Matias-Guiu X, Dolcet X. ER $\alpha$-mediated repression of pro-inflammatory cytokine expression by glucocorticoids reveals a crucial role for TNF $\alpha$ and IL1 $\alpha$ in lumen formation and maintenance. J Cell Sci 2012; 125: 1929-1944.

44. Baak JP, Kurver PH, Overdiep SH, Delemarre JF, Boon ME, Lindeman J et al. Quantitative, microscopical, computer-aided diagnosis of endometrial hyperplasia or carcinoma in individual patients. Histopathology 1981; 5: 689-695. 